\title{
Multiple Criteria Group Decision-Making Method with Dempster-Shafer Theory and Probabilistic Linguistic Term Sets
}

\author{
Yuanwei Du (D) $^{1,2}$ and Susu Wang $\mathbb{D}^{1}$ \\ ${ }^{1}$ Management College, Ocean University of China, Qingdao 266100, China \\ ${ }^{2}$ Marine Development Studies Institute of OUC, Key Research Institute of Humanities and Social Sciences at Universities, \\ Ministry of Education, Qingdao 266100, China \\ Correspondence should be addressed to Susu Wang; wangsusu@stu.ouc.edu.cn
}

Received 23 July 2020; Accepted 17 November 2020; Published 8 December 2020

Academic Editor: Zeshui Xu

Copyright (C) 2020 Yuanwei Du and SuSu Wang. This is an open access article distributed under the Creative Commons Attribution License, which permits unrestricted use, distribution, and reproduction in any medium, provided the original work is properly cited.

\begin{abstract}
The motivation of this study is to propose a novel multiple criteria group decision-making (MCDGM) method based on Dempster-Shafer theory (DST) and probabilistic linguistic term sets (PLTSs) to handle the distinctions between compensatory information at the criterion level and noncompensatory information at the individual level in the process of information fusion. Initially, the information at the individual level is extracted by BPA functions. Then, they are fused with DST considering ignorance and DMs' reliabilities. Next, the obtained BPA functions are transformed into interval-valued PLTSs with the assistance of intermediate belief and plausibility. Subsequently, the interval-valued PLTSs are converted into standard PLTSs. After normalization, the holistic PLTS is obtained with weighted addition operation and the round function is applied to determine the ultimate evaluation result. Finally, a case simulation study of evaluating the marine ranching ecological security is presented to verify and improve the validity and feasibility of the proposed method and algorithm in practical application. The proposed method and its relevant algorithm are both innovative combination of DST and PLTSs from the perspective of compensatory and noncompensatory features of information, which provides a new angle of view for the development of probabilistic preference theory and is beneficial to apply probabilistic preference theory in practice.
\end{abstract}

\section{Introduction}

Multiple criteria decision-making (MCDM) theory is composed of a series of methods that deal with alternative ranking or selection problems that are characterized by multiple attributes and conflicting noncommensurable objectives. Introduced in the 1950s [1,2], MCDM has since received extensive attention from two kinds of methods: the multiple attribute decision-making (MADM) method and the multiple objective decision-making (MODM) method. MCGDM is an indispensable branch of modern decisionmaking theory. The representative methods of MCDM, such as multiobjective programming, analytic hierarchy process (AHP), ELimination Et Choice Translating REality (ELECTRE), and technique for order preference by similarity to an ideal solution (TOPSIS), have been applied in solving diversified practical MCDM problems [3-8]. As decision-making problems are getting more complicated, researchers have studied MCDM from the perspectives of utility achievement, weight determination, consistency measurement, fuzziness information, and so on [9-13].

MCDM methods usually comprehensively analyze MCDM problems from all angles. Their applications are achieved by balancing multiple attributes or objectives to find the best one among several given alternatives. In the implementation process of classical MCDM, an individual decision maker (DM) is the single source providing the decision information that involves all the factors and forms the final decision results. However, limited by the incomplete knowledge reserve, restricted ability of judgment, inadequate experience, and other such issues, an individual DM cannot provide sufficient valid information for today's 
complex and synthetic MCDM. Consequently, multiple criteria group decision-making (MCGDM) methods [14-16] have been proposed to solve MCDM problems that arise under the group decision-making setting. The major MCGDM methods provide a basic framework for implementation that comprises extracting individual information first and then fusing it into group information. Here, two kinds of problems are of vital importance. One is about information expression and extraction, and the other is about information disposal and fusion. With respect to the former, existing literature has a lot of focus on enhancing the accuracy of the information given by each individual DM. Linguistic term, interval value, fuzzy number, membership, and other forms of information [17-20] are incorporated to facilitate DMs determining or expressing their preference. The information characteristics of uncertainty, fuzziness, and incompleteness [21-24] are also key concerns of MCGDM. With respect to the problem of information disposal and fusion, researchers have proposed several fusion methods to fuse individual information, such as the consensus model, fuzzy operators, Dempster-Shafer theory (DST), evidence reasoning (ER) approach, and probabilistic linguistic term set (PLTS) operations [25-27]. Different disposal and fusion processes usually cause different degrees of information loss, which affects the feasibility and validity of the decision results. To address this issue, several solutions, such as information conflict, reliability and weight of single information, consistency measure, and correlation measure [28-31] have been proposed in the published literature.

The motivation of this study is to propose a novel MCGDM method based on DST and PLTSs to overcome the problem of distinctions between information at different dimensions potentially disturbing the process of information fusion and thus influencing the final result of the MCGDM. These distinctions are performed since the information at the individual level is noncompensatory and that at the criterion level is compensatory in the process of information fusion. Note that MCGDM is the combination of MCDM and group decision making (GDM). In MCDM, the information at the criterion level is fused into a holistic score or value pertaining to the whole alternative, and according to this, the alternatives are ranked or selected [32]. In GDM, the final group decisions are derived through fusing information at the individual level [33]. The information involved in MCGDM is related to both the criterion and individual level. Therefore, the above distinctions should be considered carefully in MCGDM and distinct fusion rules corresponding to different information dimensions should be utilized.

In the process of implementing MCGDM, a set of criteria are selected to characterize the alternative in different aspects that are essential for the MCGDM problem [34]. For a specific MCGDM problem, the alternative always has good or poor performance (information at the criterion level) on different criteria with different weights, and these performances can compensate for each other [34-38] in additive fusion. Additive fusion is a process of trade-off where poor (good) performance on one criterion can be offset by good (poor) performance on other criteria [34] and performance on a high (low) weighted criterion can be offset by performance on a low (high) weighted criterion [36]. Consequently, the advantages or weaknesses of the alternative may not be revealed in the final comprehensive results [35]. Based on the above features, we believe that the fusion of information at the criterion level should follow compensatory strategies, which means that the holistic performance of the alternative is derived by adding performances on the criteria with equal or unequal weights [38]. In the published literature, common compensatory models are weighted additive, including but not limited to simple additive weighting (SAW), TOPSIS, AHP, and fuzzy AHP [39]. Among the existing compensatory methods, PLTSs [27] are an effective technique owing to their advantages of linguistic preference expression, probability measure, and multiple operations $[40,41]$. PLTSs and its variants have solved various practical problems. For example, the reliable participant selection problem in mobile crowdsensing has been solved by a probabilistic linguistic VIKOR method based on TODIM [42], the edge node selection problem in edge computing has been solved using the probabilistic linguistic ELECTRE II method [43], and the problem of evaluating the Internet of things platforms has been solved using an integrated probabilistic linguistic MCDM method [44].

In addition to the criteria, individual DMs constitute another dimension in the process of MCGDM. DMs in a group are invited to give judgment information based on their preference about the performance of the alternative on a single criterion, and information at the individual level is then fused into group judgment information on a single criterion or an alternative (after information fusion at the criterion level). However, limited by different degrees of bounded rationality $[38,45]$, the information given by different DMs is usually uncertain and has different degrees of reliability, which is a key factor in the fusion of information at the individual level. When a DM is definitely reliable, their judgment information is assigned total belief in the process of fusion. If a DM is not entirely reliable, their judgment information is assigned a certain degree of belief instead of total belief in the process of information fusion. In other words, the fusion result is relative to the most reliable judgment and cannot be reserved by others $[34,37,38]$. Influenced by lower reliable information, information at the individual level is incomplete, and therefore, the compensatory strategies cannot be applied in the process of fusion [38]. Hence, noncompensatory strategies are employed. Compared with the analytic cognition in compensatory strategies, noncompensatory strategies are akin to intuitive cognition [46]. Noncompensatory strategies are more suitable for explaining DMs' decision-making behavior because of their lower computational demand and dependence on the most reliable information $[36,38]$. In the published literature, classical noncompensatory models include conjunctive, disjunctive, elimination by aspects, lexicographic, and "take the best" [37]. DST [26, 47] is a superior method for noncompensatory strategies [48] because of its ability of expressing and fusing uncertain information. DST and its subsequent versions have been 
employed to solve multiple practical problems, such as mapping flood susceptibility [49], predicting rolling bearing faults [50], and determining artificial recharge location [51], among others.

To sum up, compensatory and noncompensatory strategies are two indispensable information fusion strategies that are suitable when information belongs to different dimensions. However, the published MCGDM methods have not considered the distinctions between compensatory information at the criterion level in MCDM and noncompensatory information at the individual level in GDM, which could result in compromised decision validity and quality. As MCGDM problems are getting more and more complex, it is advisable and effective to choose both type of strategies in the process of information fusion. We propose a new MCGDM method that considers both compensatory and noncompensatory strategies. The rest of this paper is organized as follows. In Section 2, we introduce the background knowledge about DST and PLTSs. In Section 3, a novel MCGDM method is proposed considering both compensatory and noncompensatory strategies based on DST and PLTSs, and the corresponding algorithm is constructed. A case simulation study of evaluating marine ranching ecological security (MRES) is presented to test the scientific validity and practical feasibility of the proposed method and algorithm in Section 4. Section 5 concludes the paper.

\section{Preliminaries}

2.1. Dempster-Shafer Theory. DST is an uncertainty reasoning technique proposed by Dempster [47] and Shafer [26]. It can handle uncertain information at the individual level on the basis of a frame of discernment, which is composed of a set of mutually exclusive and collectively exhaustive propositions. The basic probability assignment (BPA) function is applied to extract the uncertain information.

Definition 1 (see [47]). Suppose a possible proposition is $\theta_{n}(n=1,2, \ldots, N)$, and each of the proposition is exclusive. Then, a finite nonempty exhaustive set of all propositions $\Theta=\left\{\theta_{1}, \ldots, \theta_{N}\right\}$ is called a frame of discernment, and its power set that consists of $2^{N}$ subsets of $\Theta$ is usually expressed as

$$
P(\Theta)=2^{\Theta}=\left\{\varnothing, \theta_{1}, \ldots, \theta_{N},\left\{\theta_{1}, \theta_{2}\right\}, \ldots,\left\{\theta_{N-1}, \theta_{N}\right\}, \ldots,\left\{\theta_{1}, \ldots, \theta_{N-1}\right\}, \Theta\right\}
$$

Definition 2 (see [47]). Suppose $\theta$ is a nonempty subset of $\Theta$, and its belief is $p(\theta)$. If the mapping function $p: 2^{\Theta} \longrightarrow[0,1]$ fulfills

$$
\begin{cases}p(\theta)=0, & \theta=\varnothing, \\ p(\theta) \geqslant 0, \sum_{\theta \subseteq \Theta} p(\theta)=1, & \theta \neq \varnothing,\end{cases}
$$

where $\varnothing$ represents the empty set, then $p(\theta)$ is called the BPA function. If $p(\theta)>0, \theta$ is named a focal element. $p(\theta)$ is a basic probability that is assigned exactly to $\theta$ and not to any smaller subset.

Considering the situation that counterintuitive problems $[52,53]$ may impede the combination of evidences with the orthogonal sum operator in Dempster's rule, Shafer proposed a discounting method, named Shafer discounting, to solve these kinds of problems.
Definition 3 (see [26]). Suppose the belief distribution given by a piece of evidence $e_{i}$ that points to a proposition $\theta$ is $p_{\theta, i}$, and $w_{i}$ is the weight of evidence $e_{i}$ that is used to discount $p_{\theta, i}$ with $0 \leq w_{i} \leq 1, \forall i$ and $\sum_{i=1}^{I} w_{i}=1$. Then, the Shafer discounting can be defined to modify the BPA function for the evidence as follows.

$$
m_{i}= \begin{cases}w_{i} p_{\theta, i}, & \theta \subset \Theta . \\ w_{i} p_{\theta, i}+\left(1-w_{i}\right), & \theta=\Theta .\end{cases}
$$

Definition 4 (see [26]). Suppose the discounted BPA functions of two pieces of evidence $e_{1}$ and $e_{2}$ are, respectively, $m_{1}$ and $m_{2}$ on $\Theta$, and $\otimes$ is the orthogonal sum operator. For any $\theta \subseteq \Theta$, Dempster's rule is described as follows.

$$
m_{\theta, e(2)}=\left[m_{1} \otimes m_{2}\right](\theta)= \begin{cases}0, & \theta=\varnothing, \\ \frac{\sum_{\theta^{\prime} \cap \theta^{\prime \prime}=\theta, \theta^{\prime}, \theta^{\prime \prime} \subseteq \Theta} m_{\theta^{\prime}, 1} m_{\theta^{\prime \prime}, 2}}{1-\sum_{\theta^{\prime} \cap \theta^{\prime \prime}=\varnothing, \theta^{\prime}, \theta^{\prime \prime} \subseteq \Theta} m_{\theta^{\prime}, 1} m_{\theta^{\prime \prime}, 2}}, & \theta \subseteq \Theta .\end{cases}
$$

A belief measure associated with the BPA function and composed of the belief function and the plausibility function is necessary. The belief function measures the total mass that must be allocated to the elements of a certain subset of $\Theta$, and the plausibility function measures the maximal amount of mass that can be allocated to the elements of a certain subset of $\Theta$.

Definition 5 (see [47]). Suppose $m(\theta)$ is a piece of BPA function on the frame of discernment $\Theta$. Then, the belief 
function and the plausibility function of a subset $\theta$ of $\Theta$ can be denoted by

$$
\left\{\begin{array}{l}
\operatorname{Bel}(\theta)=\sum_{\theta^{\prime} \subseteq \theta} m\left(\theta^{\prime}\right), \\
\operatorname{Pl}(\theta)=\sum_{\theta^{\prime} \cap \theta \neq \varnothing} m\left(\theta^{\prime}\right),
\end{array}\right.
$$

where $\operatorname{Pl}(\theta) \geq \operatorname{Bel}(\theta)$ and $\operatorname{Pl}(\theta)=1-\operatorname{Bel}(\bar{\theta}) \cdot \bar{\theta}$ is the classical complement of $\theta$. In equation $(5), \operatorname{Bel}(\theta)$ is known as the belief function that represents the lower limit of the belief level of $\theta$, and $P l(\theta)$ is known as the plausibility function that represents the upper limit of the belief level of $\theta$. Therefore, the confidence interval $[\operatorname{Bel}(\theta), P l(\theta)]$ can describe the uncertainty about $\theta$, where $\operatorname{Bel}(\theta)$ and $P l(\theta)$, respectively, describe the minimal and maximal uncertainty about $\theta$.

2.2. Probabilistic Linguistic Term Sets. PLTSs [27] are an extension of hesitant fuzzy linguistic term sets (HFLTSs) [54] with the intention of providing a more convenient way for DMs to express their preference with probability. A series of operational laws and aggregation operations are also introduced to facilitate the fusion of information. We firstly introduce the definition of linguistic term sets (LTSs).

Definition 6 (see [55]). Let $S=\left\{s_{\alpha} \mid \alpha=0,1, \ldots, \tau\right\}$ be an LTS and $s_{\alpha}, \alpha=0,1, \ldots, \tau$ be an optional value for a linguistic variable, where $\tau$ is a positive integer that represents the total number of the linguistic terms in the LTS. Then, we have

(1) The set ordering rule as $s_{\alpha}>s_{\beta}$, if $\alpha>\beta$.

(2) The negation operator defined as neg $\left(s_{\alpha}\right)=s_{\beta}$, such that $\beta=\tau-\alpha$.

Actually, HFLTSs mainly consider the situation wherein DMs hesitate among several optional linguistic terms in the moment of making their decision. HFLTSs ignore the probabilistic messages associated with linguistic terms [27]. Therefore, PLTSs are proposed by introducing probabilistic messages in LTSs as follows.

Definition 7 (see [27]). Suppose $S=\left\{s_{\alpha} \mid \alpha=0,1, \ldots, \tau\right\}$ is an LTS. Then, its relative PLTS is denoted by

$$
L(p)=\left\{L^{(k)}\left(p^{(k)}\right) \mid L^{(k)} \in S, p^{(k)} \geq 0, k=1,2, \ldots, \# L(p), \sum_{k=1}^{\# L(p)} p^{(k)} \leq 1\right\}
$$

where $L^{(k)}\left(p^{(k)}\right)$ means that the associated probability of the $k$ th linguistic term $L^{(k)}$ in $L(p)$ is $p^{(k)}$ and $\# L(p)$ is the total number of the linguistic terms in PLTS $L(p)$. It is worth noting that $\sum_{k=1}^{\# L(p)} p^{(k)}$ represents the degree of information completeness with respect to the probabilistic distribution on all possible linguistic terms. We have $\sum_{k=1}^{\# L(p)} p^{(k)}=1$ under complete information, $\sum_{k=1}^{\# L(p)} p^{(k)}=0$ under complete ignorance, and $0<\sum_{k=1}^{\# L(p)} p^{(k)}<1$ under partial ignorance, where the ignorance represented by $1-\sum_{k=1}^{\# L(p)} p^{(k)}$ should be averagely assigned to all of the linguistic terms in $L(p)$.
The PLTSs in equation (6) are called standard PLTSs [56] with a certain point value of probability. The interval value of probability is more suitable for solving practical decisionmaking problems because of its consideration of DMs' vagueness. However, it is also less computable. Therefore, $\mathrm{Gu}$ et al. [56] provided a method for converting intervalvalued PLTSs into standard PLTSs as follows.

Definition 8 (see [56]). Suppose an interval-valued PLTS is given as $L(p)=\left\{L^{(k)}\left[l^{(k)}, u^{(k)}\right] \mid L^{(k)} \in S, 0 \leq l\right.$ $\left.(k) \leq u^{(k)} \leq 1, k=1,2, \ldots, \# L(p)\right\}$. Then, the method for converting interval-valued PLTSs into standard PLTSs is denoted by

$$
\bar{p}^{(k)}=\frac{\sum_{k=1}^{\# L(p)} u^{(k)}-1}{\sum_{k=1}^{\# L(p)} u^{(k)}-\sum_{k=1}^{\# L(p)} l^{(k)}} \times l^{(k)}+\frac{1-\sum_{k=1}^{\# L(p)} l^{(k)}}{\sum_{k=1}^{\# L(p)} u^{(k)}-\sum_{k=1}^{\# L(p)} l^{(k)}} \times u^{(k)},
$$

where $l^{(k)}$ and $u^{(k)}$ are, respectively, the lower limit and upper limit of the given interval value of probability. Obviously, standard PLTSs are special interval-valued PLTSs when $l^{(k)}=u^{(k)}$.

Certain problems are caused by the different numbers of linguistic terms in PLTSs. Therefore, an extension rule for PLTSs is introduced. The normalization of PLTSs to avoid information distortion [57] follows the rules of association and extension, as below.
Definition 9 (see [27]). If $0<\sum_{k=1}^{\# L(p)} p^{(k)}<1$, the associated PLTS $\dot{L}(p)$ of a given PLTS $L(p)$ is denoted by

$$
\dot{L}(p)=\left\{L^{(k)}\left(\dot{p}^{(k)}\right) \mid k=1,2, \ldots, \# L(p)\right\},
$$

where $\dot{p}^{(k)}=p^{(k)} / \sum_{k=1}^{\# L(p)} p^{(k)}, k=1,2, \ldots, \# L(p)$.

Definition 10 (see [27]). If $\# L_{1}(p) \neq \# L_{2}(p)$, where $\# L_{1}(p)$ and $\# L_{2}(p)$ are the numbers of linguistic terms in $L_{1}(p)$ and 
$L_{2}(p)$ respectively, suppose $L_{1}(p)$ and $L_{2}(p)$ are two PLTSs given as $L_{1}(p)=\left\{L_{1}^{(k)}\left(p_{1}^{(k)}\right) \mid k=1,2, \ldots, \# L_{1}(p)\right\}$ and $L_{2}(p)=\left\{L_{2}^{(k)}\left(p_{2}^{(k)}\right) \mid k=1,2, \ldots, \# L_{2}(p)\right\}$. Then, we should choose a PLTS with the smaller number of linguistic terms between $L_{1}(p)$ and $L_{2}(p)$ and add $\left|\# L_{1}(p)-\# L_{2}(p)\right|$ linguistic terms to it. The added linguistic terms are the smallest one(s) in the chosen PLTS, and the corresponding probability is equal to zero.

The elements in a PLTS are often disordered, which may cause operational problems. Therefore, the following ordering rule is introduced.

Definition 11 (see [27]). Given a PLTS $L(p)=\left\{L^{(k)}\left(p^{(k)}\right) \mid k=1,2, \ldots, \# L(p)\right\}$, let $r^{(k)}$ be the subscript of the linguistic term $L^{(k)}$. Then, the ordered PLTS is obtained by arranging the elements in the normalized PLTS in accordance to the values of $r^{(k)} p^{(k)}$ in descending order.

Remark 1. Considering the situation wherein two or more elements in a PLTS have equal values of $r^{(k)} p^{(k)}$, a complement for the ordering rule as in Definition 11 is introduced. Generally, when the values of $r^{(k)}$ in $r^{(k)} p^{(k)}$ are

$$
L_{1}^{N\left(k_{1}\right)}(p) \oplus L_{2}^{N\left(k_{2}\right)}(p)=\cup\left\{L_{3}^{N\left(k_{3}\right)}\left(p_{3}^{N\left(k_{3}\right)}\right) \mid k_{1}=1,2, \ldots, \# L_{1}^{N}(p), k_{2}=1,2, \ldots, \# L_{2}^{N}(p)\right\}
$$

where $L_{3}^{N\left(k_{3}\right)}=L_{1}^{N\left(k_{1}\right)} \oplus L_{2}^{N\left(k_{2}\right)}, p_{3}^{N\left(k_{3}\right)}=p_{1}^{N\left(k_{1}\right)} p_{2}^{N\left(k_{2}\right)}, L_{1}^{\left(k_{1}\right)}$ and $L_{2}^{\left(k_{2}\right)}$ are the $k_{1}$ th and $k_{2}$ th linguistic terms in $L_{1}^{N\left(k_{1}\right)}(p)$ and $L_{2}^{N\left(k_{2}\right)}(p)$, respectively, and $N$ in $L_{1}^{N\left(k_{1}\right)}$ represents that $L_{1}^{N\left(k_{1}\right)}$ is a normalized PLTS.

Virtual linguistic terms may exist when PLTSs satisfy $\lambda_{1} S_{\alpha} \oplus \lambda_{2} S_{\beta}=S_{\lambda_{1} \alpha+\lambda_{2} \beta}$. Then, the round function is as follows.

Definition 13. (see [57]). Given a PLTS $L(p)=\left\{L^{(k)}\left(p^{(k)}\right) \mid k=1,2, \ldots, \# L(p)\right\}$, the integer score (linguistic term) of $L(p)$ is denoted by

$$
E(L(p))=\operatorname{round}\left(s_{\alpha}\right)
$$

where $\alpha=\sum_{k=1}^{\# L(p)} r^{(k)} p^{(k)} / \sum_{k=1}^{\# L(p)} p^{(k)}, r^{(k)}$ is the subscript of the $k$ th linguistic term $L^{(k)}$, andround $\left(s_{\alpha}\right)$ is the classical round function that is used to determine the linguistic term that nearest to $s_{\alpha}$ with the rounding-off method.

\section{The Proposed Method}

3.1. Extraction of Information. MCGDM is usually conducted by a group of DMs who evaluate a set of given alternatives according to their own preference, with the aim to seek a satisfactory alternative or alternative rank based on a common criteria system. The only information sources in MCGDM are the DMs in the group, who may come from various fields with different degrees of knowledge reserves, experience, and cognitive styles, leading to diverse judgment unequal, the chosen elements in $L(p)$ are arranged according to the values of $r^{(k)}$ in descending order. Otherwise, the chosen elements in $L(p)$ are arranged according to the values of $p^{(k)}$ in descending order [57].

Based on the above definitions, some operations are defined as follows.

Definition 12 (see [27]). Given two ordered PLTSs $L_{1}(p)$ and $L_{2}(p)$, where $L_{1}(p)=\left\{L_{1}^{(k)}\left(p_{1}^{(k)}\right) \mid k=1,2, \ldots, \# L_{1}(p)\right\}$ and $L_{2}(p)=\left\{L_{2}^{(k)}\left(p_{2}^{(k)}\right) \mid k=1,2, \ldots, \# L_{2}(p)\right\}$, then the addition operation is defined as

$$
L_{1}(p) \oplus L_{2}(p)=\cup_{L_{1}^{(k)} \in L_{1}(p), L_{2}^{(k)} \in L_{2}(p)}\left\{p_{1}^{(k)} L_{1}^{(k)} \oplus p_{2}^{(k)} L_{2}^{(k)}\right\},
$$

where $L_{1}^{(k)}$ and $L_{2}^{(k)}$ are the $k$ th linguistic terms in $L_{1}(p)$ and $L_{2}(p)$, respectively, and the condition $\lambda_{1} S_{\alpha} \oplus \lambda_{2} S_{\beta}=S_{\lambda_{1} \alpha+\lambda_{2} \beta}$ is satisfied [58]. Then, the uniqueness of PLTSs compared with the ordinary LTSs, that is, probability, is not revealed. Zhang et al. [59] defined a new operation for normalized and ordered PLTSs as

information at the beginning of the MCGDM. The judgment information is subjectively given by DMs with different degrees of epistemic uncertainty, which could generate inaccurate decisions. Therefore, considering the uncertainty along with the preference expression of DMs is of vital importance in the extraction of information. In this regard, the BPA function in DST is an appropriate tool for extracting information, as it provides a unified way to represent preference and model uncertainty $[60,61]$.

For a specific MCGDM problem of evaluating alternatives, we give the following description. In order to solve the MCGDM problem, a group of DMs are invited to make judgment about the alternative on each given criterion. The $\mathrm{DM}$ set and criterion set are denoted by $E=\left\{e_{i} \mid i=1,2, \ldots, I\right\}$ and $C=\left\{c_{n} \mid n=1,2, \ldots, N\right\}$, where $I$ and $N$ represent the number of DMs and criteria, respectively. A set of linguistic terms denoted by $\Theta=\left\{\theta_{j} \mid j=1,2, \ldots, J\right\}$ is given for facilitating DMs to make their judgment based on their own preference with uncertainty. $\theta_{j}$ refers to the $j$ th linguistic term in the given $J$ ones. Linguistic terms in $\Theta$ are actually a series of variable values expressed in a way that conforms to human language, and each of them is mutually exclusive and exhaustive. Therefore, $\Theta$ is a finite nonempty set, called the frame of discernment, according to Definition 1. Then, the preference information given by DM $e_{i}$ on criterion $c_{n}$ can be extracted by a piece of the individual BPA function satisfying Definition 2 as 


$$
P_{i}^{n}=\left\{\left(\theta, p_{i}^{n}(\theta)\right) \mid p_{i}^{n}(\theta) \geq 0, \sum_{\theta \subseteq \Theta} p_{i}^{n}(\theta)=1, \theta \subseteq \Theta\right\},
$$

where $\theta$ is an element of the power set of the frame of discernment $P(\Theta)$ as in equation (1) and $p_{i}^{n}(\theta)$ is the probability related to $\theta$. In terms of extracting and expressing information at the individual level, the BPA function is more suitable than PLTSs to reflect the actual cognitive competence of the DMs. Since PLTSs can just reflect judgment using single linguistic term with associated probability, the BPA function additionally has the ability of presenting judgment information with local ignorance. As shown in equation (12), the value of $\theta$ describes the completeness of the DMs' recognition. When $\theta=\theta_{j}$ and $P_{i}^{n}=\left\{\left(\theta_{j}\right.\right.$, $\left.\left.p_{i}^{n}\left(\theta_{j}\right)\right) \mid p_{i}^{n}\left(\theta_{j}\right)=1\right\}$, we believe that DM $e_{i}$ has complete recognition about the alternative's performance on criterion $c_{n}$, and he/she can give a certain judgment with no ignorance [63]. When $\theta=\Theta$ and $P_{i}^{n}=\left\{\left(\Theta, p_{i}^{n}(\Theta)\right) \mid p_{i}^{n}(\Theta)=1\right\}$, it is believed that $\mathrm{DM} e_{i}$ can recognize nothing about the alternative's performance on criterion $c_{n}$, and he/she makes judgment with global ignorance [62]. $P_{i}^{n}=\{(\theta$, $\left.\left.p_{i}^{n}(\theta)\right) \mid p_{i}^{n}(\theta)=1\right\}$ means that $\mathrm{DM} e_{i}$ has incomplete recognition about the alternative's performance on criterion $c_{n}$, and he/she can make judgment with local ignorance [62]; that is, DM $e_{i}$ believes that the alternative's performance on criterion $c_{n}$ can be depicted by linguistic terms in $\theta$, but he/ she cannot decide which one in $\theta$ is the best. Equation (12) represents a more general case wherein $\mathrm{DM} e_{i}$ believes that the alternative's performance on criterion $c_{n}$ can be depicted by $\theta$ with several different values, and he/she can give relative probabilities to each of them.

3.2. Fusion of Information at the Individual Level. With the application of the BPA function, the DMs' judgment information about the alternative's performance on each criterion with corresponding probability is obtained. Then, the information at the individual level needs to be fused to get the group judgment about the alternative's performance on each criterion. It is worth noting that the BPA functions from different DMs are always associated with different degrees of uncertainty caused by objective environmental factors, subjective cognitive factors, and other factors. Even though the BPA function has abilities to express the judgments of "uncertain" or "unaware," the uncertainty caused by differences among DMs still needs to be attended. Generally, the differences among DMs are reflected in various aspects of the DMs, such as knowledge, experience, background, and authority, resulting in different degrees of reliabilities that play an important role in the process of information fusion for a particular MCGDM problem. Accordingly, the reliability of a DM is a key factor that needs to be considered in the fusion of information at the individual level.

From a group point of view, the reliability of DM $e_{i}$ actually measures the degree of influence brought by his/ her individual BPA function on the group result relative to the BPA functions given by other DMs. However, as mentioned in Section 1, this kind of influence is dominant in the process of information fusion, and influence with a low degree of reliability cannot offset that with a high degree of reliability. In other words, the information at the individual level, which is formalized as the individual BPA function, is noncompensatory, and the group result depends more on the individual BPA function given by a DM with greater reliability. For example, in the problem of evaluating the school performance of a student by three teachers, the final evaluation result is usually influenced more by (or even determined by) the teacher who knows the evaluated student more because their judgment is considered the most reliable. Suppose that the three invited teachers are $e_{1}, e_{2}$, and $e_{3}$. Teachers $e_{2}$ and $e_{3}$ just have information about the evaluated student in terms of learning and activities, and their judgments are given with local ignorance as (poor, average) and average, respectively. Teacher $e_{1}$, who has more information about the evaluated student in terms of various aspects, gives judgment as poor because he/she knows about a punishment the evaluated student has received. As a result, the final evaluation result should be poor because the judgment given by the most reliable teacher $e_{1}$ has vote power. In this example, the information given by teacher $e_{1}$ has dominant influence on the evaluation result and cannot be compensated by information given by teachers $e_{2}$ and $e_{3}$. Similarly, the judgments given by each DM in an MCGDM problem are also noncompensatory. In this scenario, DST is an effective tool for handling noncompensatory information in a more rational way [63]. In DST, an evidence with $100 \%$ reliability is totally supported; thus, it can have dominant influence on the fusion result. Otherwise, residual supports are assigned on the frame of discernment [63], which makes it possible that the residual supports are assigned on any element in the frame of discernment rather than to compensate other evidences.

Consequently, we applied DST to fuse information at the individual level. Suppose that the reliability set of DMs is $R=\left\{r_{i} \mid i=1,2, \ldots, I\right\}$. Subsequently, the extracted individual BPA functions as in equation (12) are first discounted with Shafer's discounting by taking $P_{i}^{n}$ and $R$ into equation (3). The discounted individual BPA functions can be represented as

$$
M_{i}^{n}=\left\{\left(\theta, m_{i}^{n}(\theta)\right) \mid m_{i}^{n}(\theta) \geq 0, \sum_{\theta \subseteq \Theta} m_{i}^{n}(\theta)=1, \theta \subseteq \Theta\right\},
$$

where $m_{i}^{n}(\theta)$ is the discounted probability associated with the linguistic terms in $\theta$, and we have $m_{i}^{n}(\theta)=r_{i} p_{i}^{n}(\theta)+$ $\left(1-r_{i}\right)$ when $\theta=\Theta$; otherwise, $m_{i}^{n}(\theta)=r_{i} p_{i}^{n}(\theta)$.

Next, the discounted individual BPA functions are fused with Dempster's rule by taking $M_{i}^{n}$ into equation (4). For any two given individual BPA functions from DM $e_{i}$ and $e_{i^{\prime}}$, the fusion formula is described as 


$$
m_{e(2)}^{n}(\theta)=\left[m_{i}^{n} \otimes m_{i^{\prime}}^{n}\right](\theta)= \begin{cases}0, & \theta=\varnothing, \\ \frac{\sum_{\theta^{\prime} \cap \theta^{\prime \prime}=\theta, \theta^{\prime}, \theta^{\prime \prime} \subseteq \Theta} m_{i}^{n}\left(\theta^{\prime}\right) m_{i^{\prime}}^{n}\left(\theta^{\prime \prime}\right)}{1-\sum_{\theta^{\prime} \cap \theta^{\prime \prime}=\varnothing, \theta^{\prime}, \theta^{\prime \prime} \subseteq \Theta_{i}^{n}\left(\theta^{\prime}\right) m_{i^{\prime}}^{n}\left(\theta^{\prime \prime}\right)},} & \theta \subseteq \Theta .\end{cases}
$$

Accordingly, $I$ pieces of individual BPA functions as demonstrated in equation (12) given by $\mathrm{DM} e_{1}, e_{2}, \ldots, e_{I}$ can be recursively fused into a group BPA function relative to criterion $c_{n}$. In the above process of applying DST, the reliabilities of DMs are used to discount the extracted information, where the dominant influences of DMs with higher reliabilities on the group BPA function are enhanced. Furthermore, the group judgment depends more on BPA functions with higher degrees of reliabilities under the application of the orthogonal sum operator in Dempster' rule. As a result, the influence of each individual BPA function on the group BPA function cannot compensate one another in the process of fusion, and the influences of the total reliable individual BPA functions could be entirely brought to the group BPA function with no discount. Eventually, the group BPA function relative to criterion $c_{n}$ is derived based on noncompensatory strategies as follows.

$$
M^{n}=M_{1}^{n} \otimes M_{2}^{n} \otimes \cdots \otimes M_{I}^{n}=\left\{\left(\theta, m^{n}(\theta)\right) \mid m^{n}(\theta) \geq 0, \sum_{\theta \subseteq \Theta} m^{n}(\theta)=1, \theta \subseteq \Theta\right\} .
$$

3.3. Transformation from BPA Functions into PLTSs. Based on the demonstration of DST, we have obtained $N$ pieces of group BPA functions that describe the group judgments about the alternative's performance on each criterion. In fact, the expression of information by the BPA functions facilitates handling uncertainty in DMs' judgments through assigning probabilities on the power set of the frame of discernment. When probabilities are assigned to single elements of the frame of discernment like $\theta_{j}$, we believe that the judgment is precise with no ignorance. When probabilities are assigned to a subset including more than one element like $\theta$, we believe that the judgment is "uncertain" with local ignorance. When probabilities are assigned to the frame of discernment itself like $\Theta$, we believe that the judgment is "unaware" with global ignorance. However, these kinds of data make it difficult and complicated to fuse the group judgments on each criterion. As mentioned above, the information at the criterion level, that is, the group BPA functions demonstrated by equation (15), is actually compensatory in the process of fusion. Thus, the influence given by the group BPA function on some criterion can offset that given by others in the process of fusion. Therefore, the fusion result of the information at the criterion level can reflect the comprehensive performance on all criteria and needs to be obtained with the weighted additive fusion rule. The information expression form of the BPA function is not suitable for the weighted additive fusion rule because of its assignment of probabilities. In order to establish links between information at the criterion level (group BPA functions) and the application of the weighted additive operations, the transformation method is proposed to convert the group BPA functions as in equation (15) into a simple expression appropriate for compensatory model, such as PLTSs.

In general, the probability in the BPA function usually describes the support degree of a proposition obtained from DMs. The higher the degree of support, the more certain is the proposition. In other words, the probabilities in the BPA functions are actually a kind of representation of uncertainty arising from DMs' judgments. In DST, this kind of uncertainty is measured by two functions with respect to each single linguistic term, namely, the belief function and plausibility function, which separately measure the lowest and highest probability of the proposition being true [64]. By taking the group BPA function as in equation (15) into equation (5), we can derive the belief and plausibility of the group BPA function $M^{n}$ as $\operatorname{Bel}_{n}\left(\theta_{j}\right)$ and $P l_{n}\left(\theta_{j}\right)$, which, respectively, depict the lower and upper limits of probabilities relative to single linguistic term $\theta_{j}$ on criterion $c_{n}$. From the perspective of PLTSs, $\operatorname{Bel}_{n}\left(\theta_{j}\right)$ and $P l_{n}\left(\theta_{j}\right)$ actually constitute the probability associated with linguistic term $\theta_{j}$ in the form of an interval value. For convenience, illustration, and understanding, we redefine the frame of discernment $\Theta=\left\{\theta_{j} \mid j=1,2, \ldots, J\right\}$ as an LTS $S=\left\{s_{\alpha} \mid \alpha=0,1, \ldots, \tau\right\}$ that satisfies Definition 6, where $s_{0}=\theta_{1}, s_{1}=\theta_{2}, \ldots, s_{\tau}=\theta_{J} . \operatorname{Bel}_{n}\left(\theta_{j}\right)$ and $P l_{n}\left(\theta_{j}\right)$ are redefined as $l_{n}^{(k)}$ and $u_{n}^{(k)}$, which separately represent the lower and upper limits of probability relative to the $k$ th linguistic term in LTS $S$ on criterion $c_{n}$. Accordingly, the group BPA function $M^{n}$ as in equation (15) can be transformed into a PLTS $L_{n}(p)$ as follows according to Definition 8.

$$
L_{n}(p)=\left\{L_{n}^{(k)}\left[l_{n}^{(k)}, u_{n}^{(k)}\right] \mid L_{n}^{(k)} \in S, \quad 0 \leq l_{n}^{(k)} \leq u_{n}^{(k)} \leq 1, k=1,2, \ldots, \# L_{n}(p)\right\}
$$


where $L_{n}^{(k)}$ represents the $k$ th linguistic term relative to criterion $c_{n}$ and $\# L_{n}(p)$ represents the number of linguistic terms in $L_{n}(p)$. Note that standard PLTSs are a special case of interval-valued PLTSs as in equation (16) when $l_{n}^{(k)}=u_{n}^{(k)}$.

3.4. Fusion of Information at the Criterion Level. The obtained group judgments, which are demonstrated in the form of interval-valued PLTSs on each criterion, need to be further fused to derive the holistic judgment about the whole alternative. As mentioned in Section 1, the group judgments on each criterion are actually information at the criterion level and compensatory to each other. For the whole alternative or the MCGDM problem, each criterion is selected according to a set of principles, such as systematic, typical, scientific, and comprehensive, so that the constructed criterion system can consider aspects that are integrated and valid enough for describing the MCGDM problem. In other words, each criterion in an MCGDM problem describes an essential aspect of the alternative, and the final decision is made by comprehensively combining the alternative's performance on all aspects, which is a process of trade-off. For example, in the problem of evaluating a student from the aspects of academic record $\left(c_{1}\right)$, student leader work $\left(c_{2}\right)$, and activity achievements $\left(c_{3}\right)$, bad performance on one aspect can usually be compensated by good work on other aspects because the school always train students following the principle of integrated development and any single aspect cannot decide the evaluation results. After a trade-off, a student with good performance on criterion $c_{1}$, excellent performance on criterion $c_{2}$, and poor performance on criterion $c_{3}$ may be evaluated as a good student because of the mutual compensation of performance on each aspect. Similarly, the information at the criterion level in an MCGDM problem is also compensatory. Therefore, the obtained PLTSs on each criterion as in equation (16) need to be fused into a holistic result in accordance with compensatory strategies.

As demonstrated in equation (16), the obtained PLTSs contain the information of criterion $c_{n}$, associated linguistic term $L_{n}^{(k)}$, and its corresponding interval value of probability $\left[l_{n}^{(k)}, u_{n}^{(k)}\right]$. As mentioned above, the interval value of probability is more suitable to demonstrate the vagueness of the linguistic term. However, it is not applicable for fusion operations on PLTSs. In order to solve this problem, several researchers have made strong attempts [65-67] by introducing new aggregation operators and comparison laws for interval values, which provide new calculation methods to accommodate the vagueness of linguistic terms and complicated calculations. We believe that the method as in equation (7) proposed by $\mathrm{Gu}$ et al. [56] is simple and applicable to deal with interval-valued probability. Additionally, it should be noticed that the situation of $\operatorname{Bel}_{n}\left(\theta_{j}\right)=P l_{n}\left(\theta_{j}\right)$ actually exists in practice when DMs only definitely assign the probabilities with no ignorance on single elements of the frame of discernment rather than any subset including more than one element. Thus, it is possible to have $l_{n}^{(k)}=u_{n}^{(k)}$ when the interval value of probability in the PLTSs can be regarded as a certain point value, that is, $\bar{p}_{n}^{(k)}=\left(l_{n}^{(k)}+u_{n}^{(k)}\right) / 2$. Equation (7) does not work under this circumstance. Accordingly, the obtained interval values of probabilities in the PLTSs are first converted into equivalent certain point values of probabilities as follows.

$$
\bar{p}_{n}^{(k)}= \begin{cases}\frac{l_{n}^{(k)}+u_{n}^{(k)}}{2}, & l_{n}^{(k)}=u_{n}^{(k)}, \\ \frac{\sum_{k=1}^{\# L_{n}(p)} u_{n}^{(k)}-1}{\sum_{k=1}^{\# L_{n}(p)} u_{n}^{(k)}-\sum_{k=1}^{\# L_{n}(p)} l_{n}^{(k)}} \times l_{n}^{(k)}+\frac{1-\sum_{k=1}^{\# L_{n}(p)} l_{n}^{(k)}}{\sum_{k=1}^{\# L_{n}(p)} u_{n}^{(k)}-\sum_{k=1}^{\# L_{n}(p)} l_{n}^{(k)}} \times u_{n}^{(k)}, & l_{n}^{(k)} \neq u_{n}^{(k)} .\end{cases}
$$

Hence, the interval-valued PLTSs as in equation (16) can be converted into standard PLTSs as $L_{n}(\bar{p})=\left\{L_{n}^{(k)}\right.$ $\left(\bar{p}_{n}^{(k)}\right) \mid L_{n}^{(k)} \in S, \quad \bar{p}_{n}^{(k)} \geq 0, \quad k=1,2, \ldots, \# L_{n}(\bar{p}), \sum_{k=1}^{\# L_{n}(\bar{p})}$ $\left.\bar{p}_{n}^{(k)}=1\right\}$. Afterwards, the procedure of fusing information at the criterion level can be implemented through normalization of the standard PLTSs $L_{n}(\bar{p})$. In conformity to the procedure of normalization of PLTSs, association and extension need to be carried out. However, the situation explained in Definition 9 is out of consideration here because $\# L_{n}(p)=\# L_{n}(\bar{p})$ and $\sum_{k=1}^{\# L_{n}(\bar{p})} \bar{p}_{n}^{(k)}=1$, which can be easily proven. Therefore, there is no need for association. Then, the PLTSs $L_{n}(\bar{p})$ need to be extended according to Definition 10. The probabilities assigned on the added linguistic terms are zero. Then, the extended PLTSs are arranged according to the value of $r_{n}^{(k)} \bar{p}_{n}^{(k)}$ in descending order, where $r_{n}^{(k)}$ is the subscript of linguistic term $L_{n}^{(k)}$ (see Definition 11). As a result, the normalized and ordered PLTSs are obtained as

$$
\bar{L}_{n}(\bar{p})=\left\{\bar{L}_{n}^{(k)}\left(\bar{p}_{n}^{(k)}\right) \mid \bar{L}_{n}^{(k)} \in S, \bar{p}_{n}^{(k)} \geq 0, \quad k=1,2, \ldots, \# \bar{L}_{n}(\bar{p}), \sum_{k=1}^{\# \bar{L}_{n}(\bar{p})} \bar{p}_{n}^{(k)}=1\right\}
$$


Subsequently, we need to choose an operation to derive the fusion result of information at the criterion level, where the weight of the criterion is also a necessary factor that has substantial effect on the fusion result. Therefore, we apply the redefined addition operation [19] to fuse the normalized and ordered PLTSs. Let $W=\left\{w_{n} \mid 0 t \leq n w_{n} q \leq h 1, x\right.$
$\left.\sum_{n=1}^{N} w_{n}=C 1 ;, n=1,2, \ldots, N\right\}$ be the weight set corresponding to criterion set $C=\left\{c_{n} \mid n=1,2, \ldots, N\right\}$. Then, any two given PLTSs $\bar{L}_{n}(\bar{p})$ and $\bar{L}_{n^{\prime}}(\bar{p})$ satisfy $w_{n} S_{\alpha} \oplus w_{n^{\prime}} S_{\beta}=S_{w_{n} \alpha+w, \beta}, \alpha, \beta=0,1, \ldots, \tau$. Based on Definition 12 , the weighted addition operation of any two given PLTSs $\bar{L}_{n}(\bar{p})$ and $\bar{L}_{n^{\prime}}(\bar{p})$ is as follows.

$$
w_{n} \bar{L}_{n}^{\left(k_{n}\right)}(\bar{p}) \oplus w_{n^{\prime}} \bar{L}_{n^{\prime}}^{\left(k_{n^{\prime}}\right)}(\bar{p})=\cup\left\{\bar{L}_{\delta}^{\left(k_{\delta}\right)}\left(\bar{p}_{\delta}^{\left(k_{\delta}\right)}\right) \mid \quad k_{n}=1,2, \ldots, \# \bar{L}_{n}(\bar{p}), k_{n^{\prime}}=1,2, \ldots, \# \bar{L}_{n^{\prime}}(\bar{p})\right\}
$$

where

$$
\left\{\bar{L}_{\delta}^{\left(k_{\delta}\right)}=w_{n} L_{n}^{\left(k_{n}\right)} \oplus w_{n^{\prime}} L_{n^{\prime}}^{\left(k_{n^{\prime}}\right)}, \bar{p}_{\delta}^{\left(k_{\delta}\right)}=\bar{p}_{n}^{\left(k_{n}\right)} \bar{p}_{n^{\prime}}^{\left(k_{n^{\prime}}\right)},\right.
$$

in which $\bar{L}_{\delta}^{\left(k_{\delta}\right)}, k_{\delta}=1,2, \ldots, \# \bar{L}_{\delta}(\bar{p})$ represents the $k_{\delta}$ th linguistic term in the fusion result of PLTSs $\bar{L}_{1}(\bar{p})$ and $\bar{L}_{2}(\bar{p})$. The value of $\bar{L}_{\delta}^{\left(k_{\delta}\right)}$ is calculated by $w_{n} L_{n}^{\left(k_{n}\right)} \oplus w_{n^{\prime}} L_{n^{\prime}}^{\left(k_{n^{\prime}}\right)}$, which obeys the rule $w_{n} S_{\alpha} \oplus w_{n^{\prime}} S_{\beta}=S_{w_{n} \alpha+w^{\prime},}$, $\alpha, \beta=0,1, \ldots, \tau \cdot \bar{p}_{\delta}^{\left(k_{\delta}\right)}$ is the probability relative to the linguistic term $\bar{L}_{\delta}^{\left(k_{\delta}\right)}$ in the fusion result, which is determined based on the redefined addition operation as in equation (10). For convenience of understanding, here we introduce a simple example to illustrate.

Example 1. Given two PLTSs demonstrated by equation (18) as $\quad \bar{L}_{1}(\bar{p})=\left\{\left(s_{0}, 0.1\right),\left(s_{1}, 0.3\right),\left(s_{2}, 0.6\right)\right\} \quad$ and $\bar{L}_{2}(\bar{p})=\left\{\left(s_{0}, 0.4\right),\left(s_{1}, 0.3\right),\left(s_{2}, 0.3\right)\right\}$ and their respective weights as $w_{1}=0.3$ and $w_{2}=0.7$, the calculation of fusing these two PLTSs by equations (19) and (20) is as follows.

$$
\begin{aligned}
w_{1} \bar{L}_{1}(\bar{p}) \oplus w_{2} \bar{L}_{2}(\bar{p})= & \left\{\left(s_{0.3 \times 0+0.7 \times 0}, 0.1 \times 0.4\right),\left(s_{0.3 \times 0+0.7 \times 1}, 0.1 \times 0.3\right),\left(s_{0.3 \times 0+0.7 \times 2}, 0.1 \times 0.3\right),\left(s_{0.3 \times 1+0.7 \times 0}, 0.3 \times 0.4\right)\right. \\
& \left(s_{0.3 \times 1+0.7 \times 1}, 0.3 \times 0.3\right),\left(s_{0.3 \times 1+0.7 \times 2}, 0.3 \times 0.3\right),\left(s_{0.3 \times 2+0.7 \times 0}, 0.6 \times 0.4\right),\left(s_{0.3 \times 2+0.7 \times 1}, 0.6 \times 0.3\right) \\
& \left.\left(s_{0.3 \times 2+0.7 \times 2}, 0.6 \times 0.3\right)\right\} \\
= & \left\{\left(s_{2}, 0.18\right),\left(s_{1.3}, 0.18\right),\left(s_{1.7}, 0.09\right),\left(s_{0.6}, 0.24\right),\left(s_{1}, 0.09\right),\left(s_{1.4}, 0.03\right),\left(s_{0.3}, 0.12\right),\left(s_{0.7}, 0.03\right),\left(s_{0}, 0.04\right)\right\}
\end{aligned}
$$

Subsequently, the information at the criterion level is fused into a piece of holistic PLTS relative to the whole alternative by recursively repeating the fusion as in equations (19) and (20) for $N-1$ times, where the weight of each intermediate PLTS should be determined carefully. Suppose $w_{\Delta}$ is the weight of the intermediate PLTS that is the fusion result of the first few PLTSs. Initially, for $n=1$, we have $w_{\Delta}=$ 0 and then $\widehat{L}^{(k)}=\bar{L}_{1}^{\left(k_{1}\right)}, \widehat{p}^{(k)}=\bar{p}_{1}^{\left(k_{1}\right)}$. Then, for $n=2$, we conduct the first fusion and have $w_{\Delta}=w_{1}$, and $\widehat{L}^{(k)}=w_{\Delta} \widehat{L}^{(k)} \oplus w_{2} \bar{L}_{2}^{\left(k_{2}\right)}, \quad \widehat{p}^{(k)}=\widehat{p}^{(k)} \bar{p}_{2}^{\left(k_{2}\right)}$. For $n=3$, we conduct the second fusion and have $w_{\Delta}=w_{\Delta}+w_{2}$, and $\widehat{L}^{(k)}=w_{\Delta} \widehat{L}^{(k)} \oplus w_{3} \bar{L}_{3}^{\left(k_{3}\right)}, \widehat{p}^{(k)}=\widehat{p}^{(k)} \bar{p}_{3}^{\left(k_{3}\right)}$. In general, for $\forall n=2, \ldots, N$, we have $w_{\Delta}=w_{\Delta}+w_{n-1}$, and $\widehat{L}^{(k)}=w_{\Delta} \widehat{\widehat{L}}^{(k)} \oplus w_{n} \bar{L}_{n}^{\left(k_{n}\right)}, \widehat{p}^{(k)}=\widehat{p}^{(k)} \bar{p}_{n}^{\left(k_{n}\right)}$. Finally, the obtained holistic PLTS that describes the group judgment on all criteria is denoted by

$$
\widehat{L}(\widehat{p})=w_{1} \bar{L}_{1}(\bar{p}) \oplus w_{2} \bar{L}_{2}(\bar{p}) \oplus \cdots \oplus w_{N} \bar{L}_{N}(\bar{p})=\left\{\widehat{L}^{(k)}\left(\widehat{p}^{(k)}\right) \mid \widehat{L}^{(k)} \in S, \widehat{p}^{(k)} \geq 0, k=1,2, \ldots, \# \widehat{L}(\widehat{p})\right\}
$$

Obviously, virtual linguistic terms may exist in $\widehat{L}(\widehat{p})$ (see Example 1). The ultimate result of MCGDM is calculated by taking $_{\widehat{A}} \widehat{L}(\widehat{p})$ into equation (11), and we have $\alpha=\sum_{k=1}^{\# \widehat{L}(p)} \widehat{r}^{(k)} \widehat{p}^{(k)} / \sum_{k=1}^{\# \bar{L}^{(p)}} \widehat{p}^{(k)}$, where $\widehat{r}^{(k)}$ is the subscript of the $k$ th linguistic term $\hat{\bar{L}}^{(k)}$ in holistic PLTS $\widehat{L}(\widehat{p})$, and $\widehat{p}^{(k)}$ is its associated probability. Let the ultimate result of the
MCGDM be $s^{*}$. Then, $s^{*}=\operatorname{round}\left(s_{\alpha}\right)$ is the linguistic term that is nearest to $s_{\alpha}$.

Example 2. Considering the situation that the criterion set is $C=\left\{c_{1}, c_{2}\right\}$ and the holistic PLTS is obtained as in Example 
1 , then the integer score of $\widehat{L}(\widehat{p})$ is calculated by $E(\widehat{L}(\widehat{p}))=\operatorname{round}\left(s_{\alpha}\right)$, where

$$
\alpha=\frac{0 \times 0.04+0.7 \times 0.03+1.4 \times 0.03+0.4 \times 0.12+1 \times 0.09+1.7 \times 0.09+0.6 \times 0.24+1.3 \times 0.18+2 \times 0.18}{0.04+0.03+0.03+0.12+0.09+0.09+0.24+0.18+0.18}=1.092
$$

Thus, we have $s^{*}=\operatorname{round}\left(s_{1.092}\right)=s_{1}$.

3.5. MCGDM Process and Algorithm. For convenience of illustration, the operational process of the proposed method is summarized in Figure 1. In summary, we present a novel MCGDM method using DST and PLTSs for distinguishing the noncompensatory information at the individual level and compensatory information at the criterion level in the process of information fusion. Firstly, the extraction of information based on the BPA function in DST is introduced considering ignorance that exists in DMs' judgments. Then, DST is applied to discount and obtain the group judgment on each criterion, where the reliabilities of DMs are considered. The information at the individual level is fused based on the noncompensatory strategy. In order to establish links between the group judgment formed in the group BPA function and the compensatory fusion strategy for information at the criterion level, we propose a transformation from BPA functions into PLTSs based on the belief and plausibility function in DST. Consequently, we obtain the information at the criterion level, which is in the form of interval-valued PLTSs. Thus, a method for converting interval-valued PLTSs into standard PLTSs is constructed. Finally, the standard PLTSs on each criterion are fused into a holistic PLTS with weighted addition operations, and the final MCGDM result is determined based on the round function. To facilitate practical demonstration, the above process is simplified as in the following Algorithm 1.

\section{Case Simulation Study}

In order to verify and improve the scientific validity and practical feasibility of the proposed method and algorithm, we give a case simulation study of evaluating marine ranching ecological security in this section to offer a simulative demonstration of the proposed MCGDM method. According to the definition given by the Chinese Academy of Aquatic Sciences, marine ranching is a fishery mode based on the principle of marine organisms to breed, grow bait, or avoid enemies, through releasing artificial reefs and stock enhancement. It is conductive to increase and protect fishery resources, improve the marine ecological environment, and realize sustainable utilization of fishery resources [57] (Aquatic Industry SC/T9111-2017). As a fishery mode that safeguards economic, social, and environmental revenue, marine ranching has received substantial attention in terms of various aspects, especially MRES. The objective of the case simulation study is to evaluate the MRES of a marine ranch by applying the proposed method and algorithm.
MRES is a kind of integral balance of the marine resources, marine environment, fishery activities, and other human activities. In order to keep this kind of balance, humans perform actions of releasing artificial reef and cultivating seaweed and other measures to develop a habitat for the target species. Other environmental actions are also taken to protect and improve the marine ranching ecosystem. Enhancement of fishery resources and sustainable increase of the fishery output are two main components of MRES. Consequently, we believe that the problem of evaluating MRES of a specific marine ranch includes five indispensable criteria: special funds acquisition and management $\left(c_{1}\right)$, cost and profit of the marine ranch $\left(c_{2}\right)$, monitoring system and regular inspections $\left(c_{3}\right)$, main biomass index $\left(c_{4}\right)$, and seawater and seaweed bed quality $\left(c_{5}\right)$. Suppose five DMs, namely, marine environmental monitoring technician $\left(e_{1}\right)$, aquaculture expert $\left(e_{2}\right)$, manager of the evaluated marine ranching $\left(e_{3}\right)$, marine environment and resource specialist $\left(e_{4}\right)$, and local governmental personnel $\left(e_{5}\right)$, are invited to participate in the evaluation of MRES. We denote the criterion set and DM set by $C=\left\{c_{n} \mid n=1,2, \ldots, 5\right\}$ and $E=\left\{e_{i} \mid i=1,2\right.$, $\ldots, 5\}$. Correspondingly, the weight set of the criteria and reliability set of the DMs are obtained as $W=(0.150,0.050,0.100,0.400,0.300)$ and $R=(0.680$, $0.920,0.600,1.000,0.800)$, respectively. The DMs need to make judgment about the performance of the specific marine ranch on each criterion based on the given frame of discernment that includes three linguistic terms: $\Theta=\left(\theta_{1}=\right.$ bad, $\theta_{2}=$ average, $\theta_{3}=$ good $)$.

4.1. Extracting Information from the Five DMs. Based on the above description, the individual information on each criterion given by each DM is extracted with the BPA function as in equation (12), as shown in Table 1.

It is clearly shown in Table 1 that $\mathrm{DMs} e_{1}, e_{2}, \ldots, e_{5}$ give their judgments about the MRES performance of the evaluated marine ranch on five criteria relative to the power set of frame of discernment with probabilities. For example, the extracted information given by DM $e_{1}$ on criterion $c_{1}$ can be expressed as $P_{1}^{1}=\left\{\left(\theta_{2}, 0.300\right),\left(\theta_{3}, 0.600\right),(\Theta, 0.100)\right\}$, which means that $\mathrm{DM} e_{1}$ believes that the MRES performance of the evaluated marine ranch on criterion $c_{1}$ has $30 \%$ probability to be average $\left(\theta_{2}\right), 60 \%$ probability to be good $\left(\theta_{3}\right)$, and $10 \%$ probability that the DM cannot make judgment with global ignorance. As another example, DM $e_{5}$ believes that the MRES performance of the evaluated marine ranch on criterion $c_{2}$ can be evaluated as average $\left(\theta_{2}\right)$ or good $\left(\theta_{3}\right)$; he/she only has $10 \%$ probability to hold average $\left(\theta_{2}\right)$. For the remaining $90 \%$ probability, he/she cannot 


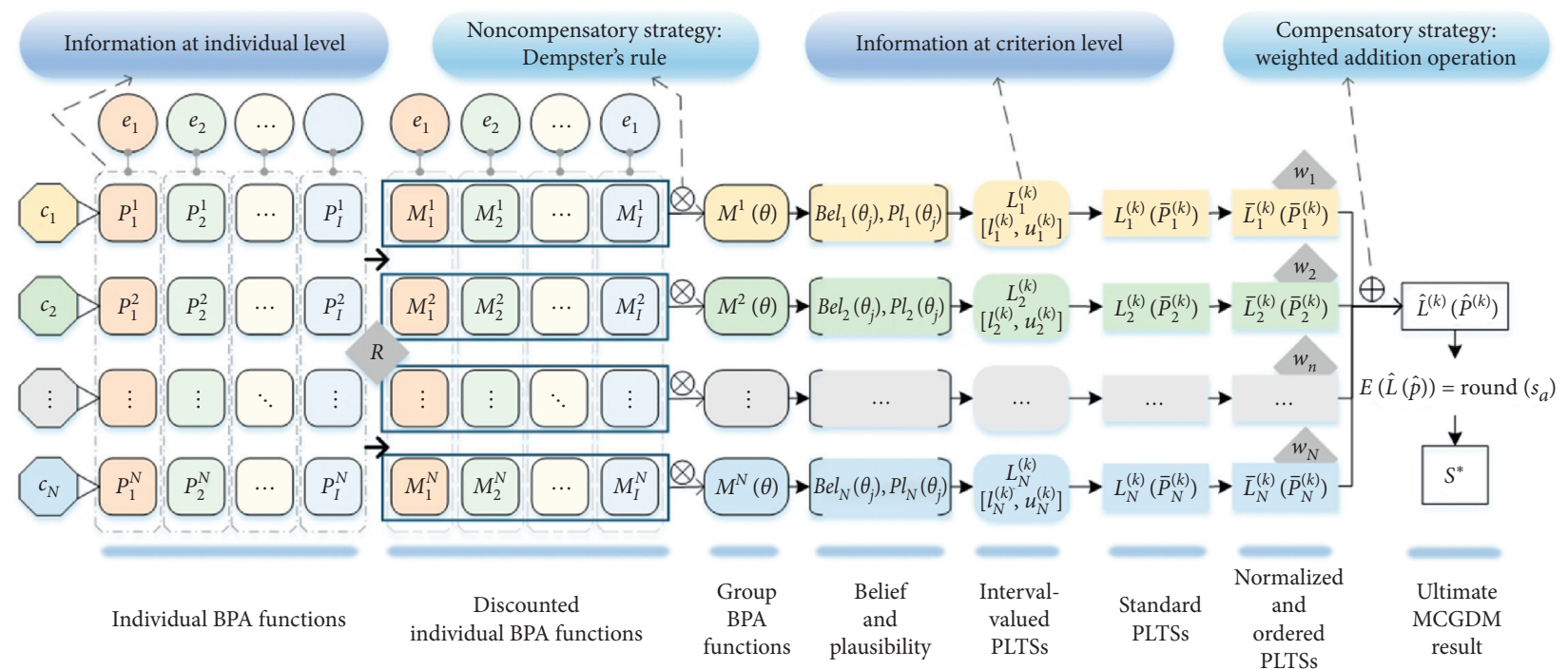

FIgURE 1: Operational process of the proposed method.

Input: extracted information from DM $e_{i}$ on criterion $c_{n}$ named $P_{i}^{n}, \forall i, n$, the DMs' reliability set $R$, and the criteria weight set $W$.

Output: the ultimate linguistic term $s^{*}$

Begin

$\%$ Fusion of information at the individual level based on noncompensatory strategies

$\%$ Discount the individual BPA functions with Shafer discounting

For $i=1$ to $I$

$$
\text { If } \theta=\Theta
$$

Then $m_{i}^{n}(\theta)=r_{i} p_{i}^{n}(\theta)+\left(1-r_{i}\right)$

Else $m_{i}^{n}(\theta)=r_{i} p_{i}^{n}(\theta)$

$$
\text { EndIf }
$$

EndFor

$\%$ Fuse the discounted individual BPA functions with Dempster's rule

For $n=1$ to $N$

If $\theta=\varnothing$

Then $m^{n}(\theta)=0$

Else $m^{n}(\theta)=m_{1}^{n}(\theta)$

For $i=2$ to $I$

$m^{n}(\theta)=\sum_{\theta^{\prime} \cap \theta^{\prime \prime}=\theta, \theta^{\prime}, \theta^{\prime \prime} \subseteq \Theta} m_{i}^{n}\left(\theta^{\prime}\right) m_{i^{\prime}}^{n}\left(\theta^{\prime \prime}\right) /\left[1-\sum_{\theta^{\prime} \cap \theta^{\prime \prime}=\varnothing, \theta^{\prime}, \theta^{\prime \prime} \subseteq \Theta} m_{i}^{n}\left(\theta^{\prime}\right) m_{i^{\prime}}^{n}\left(\theta^{\prime \prime}\right)\right]$

EndFor

EndIf

EndFor

\% Transformation from BPA function into PLTSs

$\%$ Calculate the belief and plausibility of the group BPA functions

For $n=1$ to $N$

For $j=1$ to $J$

$\operatorname{Bel}_{n}\left(\theta_{j}\right)=\sum_{\theta^{\prime} \subseteq \theta_{j}} m^{n}\left(\theta^{\prime}\right)$

$P l_{n}\left(\theta_{j}\right)=\sum_{\theta^{\prime} \cap \theta_{j} \neq \varnothing m^{n}\left(\theta^{\prime}\right)}$

EndFor

EndFor

$\%$ Define the LTS and interval-valued probabilities

For $\alpha=0$ to $\tau$

$s_{\alpha}=\theta_{j-1}$

$S=\left\{s_{\alpha} \mid \alpha=0,1, \ldots, \tau\right\} \Longleftrightarrow \Theta=\left\{\theta_{j} \mid j=1,2, \ldots, J\right\}$

For $n=1$ to $N$

$\left[l_{n}^{(k)}, u_{n}^{(k)}\right] \Longleftrightarrow\left[\operatorname{Bel}_{n}\left(\theta_{j}\right), P l_{n}\left(\theta_{j}\right)\right]$

$\%$ Form the interval-valued PLTSs

$L_{n}(p)=\left\{L_{n}^{(k)}\left[l_{n}^{(k)}, u_{n}^{(k)}\right] \mid L_{n}^{(k)} \in S, 0 \leq l_{n}^{(k)} \leq u_{n}^{(k)} \leq 1, k=1,2, \ldots, \# L_{n}(p)\right\}$

EndFor 
EndFor

$\%$ Fuse information at the criterion level based on compensatory strategies

$\%$ Convert interval-valued PLTSs into standard PLTSs

For $n=1$ to $N$

For $k=1$ to $\# L_{n}(p)$

If $l_{n}^{(k)}=u_{n}^{(k)}$
$\quad$ Then $\bar{p}_{n}^{(k)}=\left(l_{n}^{(k)}+u_{n}^{(k)}\right) / 2$

Else $\bar{p}_{n}^{(k)}=l_{n}^{(k)} \times\left[\sum_{k=1}^{\# L_{n}(p)} u_{n}^{(k)}-1\right] /\left[\sum_{k=1}^{\# L_{n}(p)} u_{n}^{(k)}-\sum_{k=1}^{\# L_{n}(p)} l_{n}^{(k)}\right]+u_{n}^{(k)} \times\left[1-\sum_{k=1}^{\# L_{n}(p)} l_{n}^{(k)}\right] /\left[\sum_{k=1}^{\# L_{n}(p)} u_{n}^{(k)}-\sum_{k=1}^{\# L_{n}(p)} l_{n}^{(k)}\right]$

EndIf

EndFor

$\%$ Extend and arrange the linguistic terms in standard PLTSs and form the normalized and ordered PLTSs

$\bar{L}_{n}(\bar{p})=\left\{\bar{L}_{n}^{(k)}\left(\bar{p}_{n}^{(k)}\right) \mid \bar{L}_{n}^{(k)} t \in n S q, h \bar{p}_{n}^{(k)} \geq x 07, C k ;=1,2, \ldots, \# \bar{L}_{n}(\bar{p}), \sum_{k=1}^{\# \bar{n}_{n}(\bar{p})} \bar{p}_{n}^{(k)}=1\right\}$

EndFor

$\%$ Fuse the normalized and ordered PLTSs with the weighted addition operation

For $n=1$

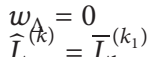

$\widehat{p}^{(k)}=\bar{p}_{1}^{\left(k_{1}\right)}$

For $n=2$ to $N$

$w_{\Delta}=w_{\Delta}+w_{n-1}$

$\widehat{L}^{(k)}=w_{\Delta} \widehat{L}^{(k)} \oplus w_{n} \bar{L}_{n}^{\left(k_{n}\right)}$

$\widehat{p}^{(k)}=\widehat{p}^{(k)} \bar{p}_{n}^{\left(k_{n}\right)}$

EndFor

EndFor

$\%$ Determine the ultimate MCGDM result

For $k=1$ to $\# L_{n}(p)$

$\alpha=\sum_{k=1}^{\# L(p)} r^{(k)} p^{(k)} / \sum_{k=1}^{\# L(p)} p^{(k)}$

$s^{*}=\operatorname{round}\left(s_{\alpha}\right)$

EndFor

End

Algorithm 1: MCGDM with DST and PLTSs.

decide which one of the two linguistic terms $\left(\left\{\theta_{2}, \theta_{3}\right\}\right)$ is the best. Accordingly, the extracted information is $P_{5}^{2}=\left\{\left(\theta_{2}, 0.100\right),\left(\left\{\theta_{2}, \theta_{3}\right\}, 0.900\right)\right\}$, which includes local ignorance. DM $e_{3}$ thinks that the MRES performance of the evaluated marine ranching on criterion $c_{1}$ can definitely be evaluated as average $\left(\theta_{2}\right)$; thus, his/her individual BPA function on criterion $c_{1}$ is $P_{3}^{1}=\left\{\left(\theta_{2}, 1.000\right)\right\}$ with no ignorance.

4.2. Fusing Information at the Individual Level. As mentioned in Section 3.2, the information given by different DMs has different degrees of reliabilities in the problem of evaluating MRES. To be specific, MRES is a complicated MCGDM problem with various aspects that are represented as the five criteria in this case. However, not every DM has enough ability, knowledge, and experience to give reliable judgment, as MRES involves many professional fields, such as government policy standards, marine sediment quality, marine environment monitoring technology, and so on. For the problem of evaluating MRES in this case, the invited marine environment and resource specialist $\left(e_{4}\right)$ is authoritative in the field of marine environmental protection and marine resource management, and his/her judgment is the most reliable in the judgments given by the five DMs. This DM's reliability is 1.000 . The marine environmental monitoring technician $\left(e_{1}\right)$, aquaculture expert $\left(e_{2}\right)$, and local governmental personnel $\left(e_{5}\right)$, respectively, have a certain degree of abilities to make judgment with reliabilities of 0.680 , 0.920 , and 0.800 . Even though the manager of the evaluated marine ranch $\left(e_{3}\right)$ knows a lot about the operation and management of the evaluated marine ranch, such as the cost and profit of the marine ranch $\left(c_{2}\right)$, he/she has the lowest reliability of 0.600 in the synthetic evaluation of the MRES. By taking the DM reliability set $R$ and the information at the individual level in Table 1 into equation (13), we obtain the discounted individual BPA functions as follows.

In the process of fusion, the information from the DM with lower reliability (e.g., $\mathrm{DM} e_{3}$ ) cannot compensate for the information from a DM with a higher reliability (e.g., DM $e_{4}$ ). So, the fusion result is effective and reliable as much as possible. Therefore, the fusion rule based on noncompensatory strategies, such as Dempster's rule, is applied, and the group BPA functions representing the group judgment of the five DMs on each criterion are obtained by taking the discounted BPA functions in Table 2 into equation (14). The obtained group BPA functions are shown in Table 3.

4.3. Transforming Group BPA Functions into PLTSs. As mentioned in Section 3.3, the BPA function provides a unified way to model and dispose the uncertainty existing in 
TABLe 1: Extracted information at the individual level.

\begin{tabular}{|c|c|c|c|c|c|c|c|c|}
\hline \multirow{2}{*}{$\mathrm{DM}$} & \multirow{2}{*}{ Criterion } & \multicolumn{7}{|c|}{ Individual probability assignment $\left(p_{i}^{n}(\theta)\right)$} \\
\hline & & $\left\{\theta_{1}\right\}$ & $\left\{\theta_{2}\right\}$ & $\left\{\theta_{3}\right\}$ & $\left\{\theta_{1}, \theta_{2}\right\}$ & $\left\{\theta_{2}, \theta_{3}\right\}$ & $\left\{\theta_{1}, \theta_{3}\right\}$ & $\Theta$ \\
\hline \multirow{5}{*}{$e_{1}$} & $c_{1}$ & 0.000 & 0.300 & 0.600 & 0.000 & 0.000 & 0.000 & 0.100 \\
\hline & $c_{2}$ & 0.000 & 0.200 & 0.500 & 0.000 & 0.200 & 0.000 & 0.100 \\
\hline & $c_{3}$ & 0.000 & 0.300 & 0.000 & 0.150 & 0.550 & 0.000 & 0.000 \\
\hline & $c_{4}$ & 0.600 & 0.000 & 0.200 & 0.000 & 0.200 & 0.000 & 0.000 \\
\hline & $c_{5}$ & 0.600 & 0.000 & 0.000 & 0.000 & 0.000 & 0.000 & 0.400 \\
\hline \multirow{5}{*}{$e_{2}$} & $c_{1}$ & 0.000 & 0.600 & 0.000 & 0.000 & 0.350 & 0.000 & 0.050 \\
\hline & $c_{2}$ & 0.000 & 0.000 & 0.300 & 0.000 & 0.000 & 0.000 & 0.700 \\
\hline & $c_{3}$ & 0.000 & 0.000 & 0.400 & 0.000 & 0.400 & 0.000 & 0.200 \\
\hline & $c_{4}$ & 0.650 & 0.150 & 0.200 & 0.000 & 0.000 & 0.000 & 0.000 \\
\hline & $c_{5}$ & 0.000 & 0.500 & 0.500 & 0.000 & 0.000 & 0.000 & 0.000 \\
\hline \multirow{5}{*}{$e_{3}$} & $c_{1}$ & 0.000 & 1.000 & 0.000 & 0.000 & 0.000 & 0.000 & 0.000 \\
\hline & $c_{2}$ & 0.000 & 0.400 & 0.600 & 0.000 & 0.000 & 0.000 & 0.000 \\
\hline & $c_{3}$ & 0.000 & 0.200 & 0.200 & 0.600 & 0.000 & 0.000 & 0.000 \\
\hline & $c_{4}$ & 0.000 & 0.300 & 0.400 & 0.300 & 0.000 & 0.000 & 0.000 \\
\hline & $c_{5}$ & 0.000 & 0.000 & 0.300 & 0.300 & 0.000 & 0.000 & 0.400 \\
\hline \multirow{5}{*}{$e_{4}$} & $c_{1}$ & 0.300 & 0.000 & 0.000 & 0.400 & 0.000 & 0.000 & 0.300 \\
\hline & $c_{2}$ & 0.000 & 0.000 & 0.000 & 0.000 & 0.000 & 0.000 & 1.000 \\
\hline & $c_{3}$ & 0.000 & 0.200 & 0.200 & 0.600 & 0.000 & 0.000 & 0.000 \\
\hline & $c_{4}$ & 0.000 & 0.000 & 0.300 & 0.000 & 0.000 & 0.000 & 0.700 \\
\hline & $c_{5}$ & 0.300 & 0.700 & 0.000 & 0.000 & 0.000 & 0.000 & 0.000 \\
\hline \multirow{5}{*}{$e_{5}$} & $c_{1}$ & 0.000 & 0.200 & 0.200 & 0.600 & 0.000 & 0.000 & 0.000 \\
\hline & $c_{2}$ & 0.000 & 0.100 & 0.000 & 0.000 & 0.900 & 0.000 & 0.000 \\
\hline & $c_{3}$ & 0.100 & 0.000 & 0.000 & 0.900 & 0.000 & 0.000 & 0.000 \\
\hline & $c_{4}$ & 0.000 & 0.200 & 0.800 & 0.000 & 0.000 & 0.000 & 0.000 \\
\hline & $c_{5}$ & 0.000 & 0.000 & 0.500 & 0.000 & 0.000 & 0.000 & 0.500 \\
\hline
\end{tabular}

DMs' judgments. As is shown in Table 3, the group BPA function on criterion $c_{1}$ is $M^{1}=\left\{\left(\theta_{1}, 0.011\right),\left(\theta_{2}, 0.924\right)\right.$, $\left.\left(\theta_{3}, 0.031\right), \quad\left(\left\{\theta_{1}, \theta_{2}\right\}, 0.022\right),\left(\left\{\theta_{2}, \theta_{3}\right\}, 0.008\right),(\Theta, 0.003)\right\}$, which contains both local ignorance and global ignorance of the DM group. The group BPA function on criterion $c_{5}$ is $M^{5}=\left\{\left(\theta_{1}, 0.097\right),\left(\theta_{2}, 0.903\right)\right\}$, which assigns the probabilities to single elements with no ignorance. Irrespective of the kind of ignorance contained, all information in the group BPA functions is associated with a degree of uncertainty, which is measured by the belief and plausibility function in DST. Therefore, we calculate the belief and plausibility of the group BPA functions by taking the group BPA functions in Table 3 into equation (5). The calculation result is provided in Table 4.

As a result, we obtain the lower and upper limits of the probability assigned to each linguistic term. For convenience of illustration and understandability, we redefine the LTS and its associated value limit of probability. According to the explanation in Section 3.3, the frame of discernment $\Theta=\left(\theta_{1}=\right.$ bad, $\theta_{2}=$ average, $\theta_{3}=$ good) is redefined as an LTS $S=\left(s_{0}=\right.$ bad, $s_{1}=$ average, $s_{2}=$ good $)$, and the associated value limit of the probability is redefined each to each. For example, the belief $\left(\operatorname{Bel}_{2}\left(\theta_{1}\right)\right)$ and plausibility $\left(P l_{2}\left(\theta_{1}\right)\right)$ of the group BPA function $M^{2}$ relative to linguistic term $\theta_{1}$ are redefined as $l_{2}^{(1)}$ and $u_{2}^{(1)}$, respectively. Accordingly, the group BPA functions in Table 3 are transformed into interval-valued PLTSs as shown in Table 5.
4.4. Fusing Information at the Criterion Level. Based on the above procedure, we obtain the interval-valued PLTSs on each criterion, which describe the possible linguistic terms and associated probabilities. For example, the interval-valued PLTS on criterion $c_{1}$ is $L_{1}(p)=\left\{s_{0}[0.011,0.037], s_{1}[0.924,0.958], s_{2}[0.031,0.042]\right\}$, which represents that the MRES performance on special funds acquisition and management $\left(c_{1}\right)$ situation of the evaluated marine ranch is possible to be evaluated as bad $\left(s_{0}\right)$,average $\left(s_{1}\right)$, and good $\left(s_{2}\right)$. Correspondingly, the associated possibilities are, respectively, between intervals $[1.1 \%, 3.7 \%],[92.4 \%, 95.8 \%]$, and [3.1\%, 4.2\%]. Subsequently, the five interval-valued PLTSs need to be fused to get the holistic PLTS. We should convert the interval-valued PLTSs into standard PLTSs with certain point-valued probabilities first so that the compensatory operations of PLTSs are applicable. By taking the interval-valued PLTSs in Table 5 into equation (17), we derive the standard PLTSs. After normalization and arrangement, the normalized and ordered PLTSs are obtained (Table 6), where $r_{n}^{(k)}$ is the subscript of the $k$ th linguistic term in the normalized and ordered PLTSs and $r_{n}^{(k)} \bar{p}_{n}^{(k)}$ is the value that is used to arrange the linguistic terms in descending order.

Generally, each PLTS in Table 6 describes the information at the criterion level. As illustrated in Section 3.4, each criterion of MRES is an independent and indispensable aspect, and all criteria compose the MCGDM problem jointly. In this case, the evaluation of MRES includes five criteria, and the information at the criterion level is 
TABLE 2: The discounted individual BPA functions.

\begin{tabular}{|c|c|c|c|c|c|c|c|c|}
\hline \multirow{2}{*}{$\mathrm{DM}$} & \multirow{2}{*}{ Criterion } & \multicolumn{7}{|c|}{ Discounted individual probability assignment $\left(m_{i}^{n}(\theta)\right)$} \\
\hline & & $\left\{\theta_{1}\right\}$ & $\left\{\theta_{2}\right\}$ & $\left\{\theta_{3}\right\}$ & $\left\{\theta_{1}, \theta_{2}\right\}$ & $\left\{\theta_{2}, \theta_{3}\right\}$ & $\left\{\theta_{1}, \theta_{3}\right\}$ & $\Theta$ \\
\hline \multirow{5}{*}{$e_{1}$} & $c_{1}$ & 0.000 & 0.204 & 0.408 & 0.000 & 0.000 & 0.000 & 0.388 \\
\hline & $c_{2}$ & 0.000 & 0.136 & 0.340 & 0.000 & 0.136 & 0.000 & 0.388 \\
\hline & $c_{3}$ & 0.000 & 0.204 & 0.000 & 0.102 & 0.374 & 0.000 & 0.320 \\
\hline & $c_{4}$ & 0.408 & 0.000 & 0.136 & 0.000 & 0.136 & 0.000 & 0.320 \\
\hline & $c_{5}$ & 0.408 & 0.000 & 0.000 & 0.000 & 0.000 & 0.000 & 0.592 \\
\hline \multirow{5}{*}{$e_{2}$} & $c_{1}$ & 0.000 & 0.552 & 0.000 & 0.000 & 0.322 & 0.000 & 0.126 \\
\hline & $c_{2}$ & 0.000 & 0.000 & 0.276 & 0.000 & 0.000 & 0.000 & 0.724 \\
\hline & $c_{3}$ & 0.000 & 0.000 & 0.368 & 0.000 & 0.368 & 0.000 & 0.264 \\
\hline & $c_{4}$ & 0.598 & 0.138 & 0.184 & 0.000 & 0.000 & 0.000 & 0.080 \\
\hline & $c_{5}$ & 0.000 & 0.460 & 0.460 & 0.000 & 0.000 & 0.000 & 0.080 \\
\hline \multirow{5}{*}{$e_{3}$} & $c_{1}$ & 0.000 & 0.600 & 0.000 & 0.000 & 0.000 & 0.000 & 0.400 \\
\hline & $c_{2}$ & 0.000 & 0.240 & 0.360 & 0.000 & 0.000 & 0.000 & 0.400 \\
\hline & $c_{3}$ & 0.000 & 0.120 & 0.120 & 0.360 & 0.000 & 0.000 & 0.400 \\
\hline & $c_{4}$ & 0.000 & 0.180 & 0.240 & 0.180 & 0.000 & 0.000 & 0.400 \\
\hline & $c_{5}$ & 0.000 & 0.000 & 0.180 & 0.180 & 0.000 & 0.000 & 0.640 \\
\hline \multirow{5}{*}{$e_{4}$} & $c_{1}$ & 0.300 & 0.000 & 0.000 & 0.400 & 0.000 & 0.000 & 0.300 \\
\hline & $c_{2}$ & 0.000 & 0.000 & 0.000 & 0.000 & 0.000 & 0.000 & 1.000 \\
\hline & $c_{3}$ & 0.000 & 0.200 & 0.200 & 0.600 & 0.000 & 0.000 & 0.000 \\
\hline & $c_{4}$ & 0.000 & 0.000 & 0.300 & 0.000 & 0.000 & 0.000 & 0.700 \\
\hline & $c_{5}^{4}$ & 0.300 & 0.700 & 0.000 & 0.000 & 0.000 & 0.000 & 0.000 \\
\hline \multirow{5}{*}{$e_{5}$} & $c_{1}$ & 0.000 & 0.160 & 0.160 & 0.480 & 0.000 & 0.000 & 0.200 \\
\hline & $c_{2}$ & 0.000 & 0.080 & 0.000 & 0.000 & 0.720 & 0.000 & 0.200 \\
\hline & $c_{3}$ & 0.080 & 0.000 & 0.000 & 0.720 & 0.000 & 0.000 & 0.200 \\
\hline & $c_{4}$ & 0.000 & 0.160 & 0.640 & 0.000 & 0.000 & 0.000 & 0.200 \\
\hline & $c_{5}$ & 0.000 & 0.000 & 0.400 & 0.000 & 0.000 & 0.000 & 0.600 \\
\hline
\end{tabular}

TABLE 3: The group BPA functions.

\begin{tabular}{|c|c|c|c|c|c|c|c|}
\hline \multirow[b]{2}{*}{ Criterion } & \multicolumn{7}{|c|}{ Group probability assignment $\left(m^{n}(\theta)\right)$} \\
\hline & $\left\{\theta_{1}\right\}$ & $\left\{\theta_{2}\right\}$ & $\left\{\theta_{3}\right\}$ & $\left\{\theta_{1}, \theta_{2}\right\}$ & $\left\{\theta_{2}, \theta_{3}\right\}$ & $\left\{\theta_{1}, \theta_{3}\right\}$ & $\Theta$ \\
\hline$c_{1}$ & 0.011 & 0.924 & 0.031 & 0.022 & 0.008 & 0.000 & 0.003 \\
\hline$c_{2}$ & 0.000 & 0.216 & 0.603 & 0.000 & 0.152 & 0.000 & 0.029 \\
\hline$c_{3}$ & 0.009 & 0.848 & 0.034 & 0.109 & 0.000 & 0.000 & 0.000 \\
\hline$c_{4}$ & 0.273 & 0.118 & 0.590 & 0.005 & 0.004 & 0.000 & 0.010 \\
\hline$c_{5}$ & 0.097 & 0.903 & 0.000 & 0.000 & 0.000 & 0.000 & 0.000 \\
\hline
\end{tabular}

TABLE 4: The belief and plausibility of the group BPA functions.

\begin{tabular}{|c|c|c|c|c|c|c|}
\hline \multirow{2}{*}{ Criterion } & \multicolumn{6}{|c|}{ Belief and plausibility of the group BPA functions $\left(\operatorname{Bel}_{n}\left(\theta_{j}\right) \& P l_{n}\left(\theta_{j}\right)\right)$} \\
\hline & $\operatorname{Bel}_{n}\left(\theta_{1}\right)$ & $P l_{n}\left(\theta_{1}\right)$ & $\operatorname{Bel}_{n}\left(\theta_{2}\right)$ & $P l_{n}\left(\theta_{2}\right)$ & $\operatorname{Bel}_{n}\left(\theta_{3}\right)$ & $P l_{n}\left(\theta_{3}\right)$ \\
\hline$c_{1}$ & 0.011 & 0.037 & 0.924 & 0.958 & 0.031 & 0.042 \\
\hline$c_{2}$ & 0.000 & 0.029 & 0.216 & 0.397 & 0.603 & 0.784 \\
\hline$c_{3}$ & 0.009 & 0.119 & 0.848 & 0.957 & 0.034 & 0.034 \\
\hline$c_{4}$ & 0.273 & 0.288 & 0.118 & 0.137 & 0.590 & 0.604 \\
\hline$c_{5}$ & 0.097 & 0.097 & 0.903 & 0.903 & 0.000 & 0.000 \\
\hline
\end{tabular}

TABle 5: The interval-valued PLTSs.

\begin{tabular}{lccc}
\hline Criterion & & Interval value of probability $\left(\left[l_{n}^{(k)}, u_{n}^{(k)}\right]\right)$ & $L_{n}^{(3)}\left[l_{n}^{(3)}, u_{n}^{(3)}\right]$ \\
\hline$c_{1}$ & $L_{n}^{(1)}\left[l_{n}^{(1)}, u_{n}^{(1)}\right]$ & $L_{n}^{(2)}\left[l_{n}^{(2)}, u_{n}^{(2)}\right]$ & $s_{2}[0.031,0.042]$ \\
$c_{2}$ & $s_{0}[0.011,0.037]$ & $s_{1}[0.924,0.958]$ & $s_{2}[0.603,0.784]$ \\
$c_{3}$ & $s_{0}[0.000,0.029]$ & $s_{1}[0.216,0.397]$ & $s_{2}[0.034,0.034]$ \\
$c_{4}$ & $s_{0}[0.009,0.119]$ & $s_{1}[0.848,0.957]$ & $s_{2}[0.590,0.604]$ \\
$c_{5}$ & $s_{0}[0.273,0.288]$ & $s_{1}[0.118,0.137]$ & - \\
\hline
\end{tabular}


TABle 6: The normalized and ordered PLTSs.

\begin{tabular}{|c|c|c|c|c|c|c|c|c|c|}
\hline \multirow{2}{*}{ Criterion } & \multicolumn{9}{|c|}{ Probability and associated value $\left(r_{n}^{(k)} \bar{p}_{n}^{(k)}\right)$} \\
\hline & $\bar{L}_{n}^{(1)}$ & $\bar{p}_{n}^{(1)}$ & $r_{n}^{(1)} \bar{p}_{n}^{(1)}$ & $\bar{L}_{n}^{(2)}$ & $\bar{p}_{n}^{(2)}$ & $r_{n}^{(2)} \bar{p}_{n}^{(2)}$ & $\bar{L}_{n}^{(3)}$ & $\bar{p}_{n}^{(3)}$ & $r_{n}^{(3)} \bar{p}_{n}^{(3)}$ \\
\hline$c_{1}$ & $s_{1}$ & 0.940 & 0.940 & $s_{2}$ & 0.036 & 0.072 & $s_{0}$ & 0.023 & 0.000 \\
\hline$c_{2}$ & $s_{2}$ & 0.687 & 1.374 & $s_{1}$ & 0.300 & 0.300 & $s_{0}$ & 0.014 & 0.000 \\
\hline$c_{3}$ & $s_{1}$ & 0.902 & 0.902 & $s_{2}$ & 0.034 & 0.068 & $s_{0}$ & 0.064 & 0.000 \\
\hline$c_{4}$ & $s_{2}$ & 0.595 & 1.190 & $s_{1}$ & 0.125 & 0.125 & $s_{0}$ & 0.279 & 0.000 \\
\hline$c_{5}$ & $s_{1}$ & 0.903 & 0.903 & $s_{0}$ & 0.097 & 0.000 & $s_{0}$ & 0.000 & 0.000 \\
\hline
\end{tabular}

TABle 7: The holistic PLTS.

\begin{tabular}{|c|c|c|}
\hline \multirow{2}{*}{ Linguistic term } & \multicolumn{2}{|c|}{ Probability assignment } \\
\hline & $\widehat{r}^{(k)}$ & $\widehat{p}^{(k)}$ \\
\hline$\widehat{L}^{(1)}$ & 0.0000 & 0.0000005 \\
\hline$\widehat{L}^{(2)}$ & 0.0021 & 0.0000121 \\
\hline$\widehat{L}^{(3)}$ & 0.0042 & 0.0000276 \\
\hline$\widehat{L}^{(4)}$ & 0.0063 & 0.0000220 \\
\hline$\widehat{L}^{(5)}$ & 0.0084 & 0.0004887 \\
\hline$\widehat{L}^{(6)}$ & 0.0105 & 0.0011203 \\
\hline$\widehat{L}^{(7)}$ & 0.0126 & 0.0000009 \\
\hline$\widehat{L}^{(8)}$ & 0.0147 & 0.0000189 \\
\hline$\widehat{L}^{(9)}$ & 0.0168 & 0.0000434 \\
\hline$\widehat{L}^{(10)}$ & 0.0210 & 0.0000076 \\
\hline$\widehat{L}^{(11)}$ & 0.0231 & 0.0001697 \\
\hline$\widehat{L}^{(12)}$ & 0.0252 & 0.0003890 \\
\hline$\widehat{L}^{(13)}$ & 0.0273 & 0.0003098 \\
\hline$\widehat{L}^{(14)}$ & 0.0294 & 0.0068758 \\
\hline$\widehat{L}^{(15)}$ & 0.0315 & 0.0157618 \\
\hline$\widehat{L}^{(16)}$ & 0.0336 & 0.0000120 \\
\hline$\widehat{L}^{(17)}$ & 0.0357 & 0.0002661 \\
\hline$\widehat{L}^{(18)}$ & 0.0378 & 0.0006101 \\
\hline$\widehat{L}^{(19)}$ & 0.0420 & 0.0000003 \\
\hline$\widehat{L}^{(20)}$ & 0.0441 & 0.0000063 \\
\hline$\widehat{L}^{(21)}$ & 0.0462 & 0.0000145 \\
\hline$\widehat{L}^{(22)}$ & 0.0483 & 0.0000116 \\
\hline$\widehat{L}^{(23)}$ & 0.0504 & 0.0002572 \\
\hline$\widehat{L}^{(24)}$ & 0.0525 & 0.0005895 \\
\hline$\widehat{L}^{(25)}$ & 0.0546 & 0.0000004 \\
\hline$\widehat{L}^{(26)}$ & 0.0567 & 0.0000100 \\
\hline$\widehat{L}^{(27)}$ & 0.0588 & 0.0000228 \\
\hline$\widehat{L}^{(28)}$ & 0.2800 & 0.0000002 \\
\hline$\widehat{L}^{(29)}$ & 0.2821 & 0.0000054 \\
\hline$\widehat{L}^{(30)}$ & 0.2842 & 0.0000124 \\
\hline$\widehat{L}^{(31)}$ & 0.2863 & 0.0000099 \\
\hline$\widehat{L}^{(32)}$ & 0.2884 & 0.0002192 \\
\hline$\widehat{L}^{(33)}$ & 0.2905 & 0.0005025 \\
\hline$\widehat{L}^{(34)}$ & 0.2926 & 0.0000004 \\
\hline$\widehat{L}^{(35)}$ & 0.2947 & 0.0000085 \\
\hline$\widehat{L}^{(36)}$ & 0.2968 & 0.0000194 \\
\hline$\widehat{L}^{(37)}$ & 0.3000 & 0.0000051 \\
\hline$\widehat{L}^{(38)}$ & 0.3010 & 0.0000034 \\
\hline$\widehat{L}^{(39)}$ & 0.3021 & 0.0001125 \\
\hline$\widehat{L}^{(40)}$ & 0.3031 & 0.0000761 \\
\hline$\widehat{L}^{(41)}$ & 0.3042 & 0.0002578 \\
\hline$\widehat{L}^{(42)}$ & 0.3052 & 0.0001745 \\
\hline$\widehat{L}^{(43)}$ & 0.3063 & 0.0002053 \\
\hline$\widehat{L}^{(44)}$ & 0.3073 & 0.0001389 \\
\hline$\widehat{L}^{(45)}$ & 0.3084 & 0.0045567 \\
\hline$\widehat{L}^{(46)}$ & 0.3094 & 0.0030839 \\
\hline$\widehat{L}^{(47)}$ & 0.3105 & 0.0104456 \\
\hline$\widehat{L}^{(48)}$ & 0.3115 & 0.0070694 \\
\hline$\widehat{L}^{(49)}$ & 0.3126 & 0.0000079 \\
\hline
\end{tabular}

TABle 7: Continued.

\begin{tabular}{|c|c|c|}
\hline$\widehat{L}^{(50)}$ & 0.3136 & 0.0000054 \\
\hline$\widehat{L}^{(51)}$ & 0.3147 & 0.0001764 \\
\hline$\widehat{L}^{(52)}$ & 0.3157 & 0.0001194 \\
\hline$\widehat{L}^{(53)}$ & 0.3168 & 0.0004043 \\
\hline$\widehat{L}^{(54)}$ & 0.3178 & 0.0002736 \\
\hline$\widehat{L}^{(55)}$ & 0.3210 & 0.0000713 \\
\hline$\widehat{L}^{(56)}$ & 0.3220 & 0.0000001 \\
\hline$\widehat{L}^{(57)}$ & 0.3231 & 0.0015821 \\
\hline$\widehat{L}^{(58)}$ & 0.3241 & 0.0000028 \\
\hline$\widehat{L}^{(59)}$ & 0.3252 & 0.0036268 \\
\hline$\widehat{L}^{(60)}$ & 0.3262 & 0.0000065 \\
\hline$\widehat{L}^{(61)}$ & 0.3273 & 0.0028885 \\
\hline$\widehat{L}^{(62)}$ & 0.3283 & 0.0000052 \\
\hline$\widehat{L}^{(63)}$ & 0.3294 & 0.0641101 \\
\hline$\widehat{L}^{(64)}$ & 0.3304 & 0.0001153 \\
\hline$\widehat{L}^{(65)}$ & 0.3315 & 0.1469629 \\
\hline$\widehat{L}^{(66)}$ & 0.3325 & 0.0002644 \\
\hline$\widehat{L}^{(67)}$ & 0.3336 & 0.0001118 \\
\hline$\widehat{L}^{(68)}$ & 0.3346 & 0.0000002 \\
\hline$\widehat{L}^{(69)}$ & 0.3357 & 0.0024815 \\
\hline$\widehat{L}^{(70)}$ & 0.3367 & 0.0000045 \\
\hline$\widehat{L}^{(71)}$ & 0.3378 & 0.0056885 \\
\hline$\widehat{L}^{(72)}$ & 0.3388 & 0.0000102 \\
\hline$\widehat{L}^{(73)}$ & 0.3420 & 0.0000027 \\
\hline$\widehat{L}^{(74)}$ & 0.3441 & 0.0000592 \\
\hline$\widehat{L}^{(75)}$ & 0.3462 & 0.0001356 \\
\hline$\widehat{L}^{(76)}$ & 0.3483 & 0.0001080 \\
\hline$\widehat{L}^{(77)}$ & 0.3504 & 0.0023977 \\
\hline$\widehat{L}^{(78)}$ & 0.3525 & 0.0054965 \\
\hline$\widehat{L}^{(79)}$ & 0.3546 & 0.0000042 \\
\hline$\widehat{L}^{(80)}$ & 0.3567 & 0.0000928 \\
\hline$\widehat{L}^{(81)}$ & 0.3588 & 0.0002128 \\
\hline$\widehat{L}^{(82)}$ & 0.5600 & 0.0000012 \\
\hline$\widehat{L}^{(83)}$ & 0.5621 & 0.0000257 \\
\hline$\widehat{L}^{(84)}$ & 0.5642 & 0.0000590 \\
\hline$\widehat{L}^{(85)}$ & 0.5663 & 0.0000470 \\
\hline$\widehat{L}^{(86)}$ & 0.5684 & 0.0010422 \\
\hline$\widehat{L}^{(87)}$ & 0.5705 & 0.0023890 \\
\hline$\widehat{L}^{(88)}$ & 0.5726 & 0.0000018 \\
\hline$\widehat{L}^{(89)}$ & 0.5747 & 0.0000403 \\
\hline$\widehat{L}^{(90)}$ & 0.5768 & 0.0000925 \\
\hline$\widehat{L}^{(91)}$ & 0.5800 & 0.0000023 \\
\hline$\widehat{L}^{(92)}$ & 0.5810 & 0.0000163 \\
\hline$\widehat{L}^{(93)}$ & 0.5821 & 0.0000504 \\
\hline$\widehat{L}^{(94)}$ & 0.5831 & 0.0003618 \\
\hline$\widehat{L}^{(95)}$ & 0.5842 & 0.0001156 \\
\hline$\widehat{L}^{(96)}$ & 0.5852 & 0.0008295 \\
\hline$\widehat{L}^{(97)}$ & 0.5863 & 0.0000921 \\
\hline$\widehat{L}^{(98)}$ & 0.5873 & 0.0006606 \\
\hline$\widehat{L}^{(99)}$ & 0.5884 & 0.0020438 \\
\hline$\widehat{L}^{(100)}$ & 0.5894 & 0.0146624 \\
\hline
\end{tabular}


TABLE 7: Continued.

\begin{tabular}{|c|c|c|}
\hline$\widehat{L}^{(101)}$ & 0.5905 & 0.0046850 \\
\hline$\widehat{L}^{(102)}$ & 0.5915 & 0.0336114 \\
\hline$\widehat{L}^{(103)}$ & 0.5926 & 0.0000036 \\
\hline$\widehat{L}^{(104)}$ & 0.5936 & 0.0000256 \\
\hline$\widehat{L}^{(105)}$ & 0.5947 & 0.0000791 \\
\hline$\widehat{L}^{(106)}$ & 0.5957 & 0.0005675 \\
\hline$\widehat{L}^{(107)}$ & 0.5968 & 0.0001813 \\
\hline$\widehat{L}^{(108)}$ & 0.5978 & 0.0013010 \\
\hline$\widehat{L}^{(109)}$ & 0.6010 & 0.0000320 \\
\hline$\widehat{L}^{(110)}$ & 0.6020 & 0.0000006 \\
\hline$\widehat{L}^{(111)}$ & 0.6031 & 0.0007096 \\
\hline$\widehat{L}^{(112)}$ & 0.6041 & 0.0000135 \\
\hline$\widehat{L}^{(113)}$ & 0.6052 & 0.0016267 \\
\hline$\widehat{L}^{(114)}$ & 0.6062 & 0.0000310 \\
\hline$\widehat{L}^{(115)}$ & 0.6073 & 0.0012955 \\
\hline$\widehat{L}^{(116)}$ & 0.6083 & 0.0000247 \\
\hline$\widehat{L}^{(117)}$ & 0.6094 & 0.0287542 \\
\hline$\widehat{L}^{(118)}$ & 0.6104 & 0.0005484 \\
\hline$\widehat{L}^{(119)}$ & 0.6115 & 0.0659149 \\
\hline$\widehat{L}^{(120)}$ & 0.6125 & 0.0012571 \\
\hline$\widehat{L}^{(121)}$ & 0.6136 & 0.0000501 \\
\hline$\widehat{L}^{(122)}$ & 0.6146 & 0.0000010 \\
\hline$\widehat{L}^{(123)}$ & 0.6157 & 0.0011130 \\
\hline$\widehat{L}^{(124)}$ & 0.6167 & 0.0000212 \\
\hline$\widehat{L}^{(125)}$ & 0.6178 & 0.0025514 \\
\hline$\widehat{L}^{(126)}$ & 0.6188 & 0.0000487 \\
\hline$\widehat{L}^{(127)}$ & 0.6220 & 0.0000012 \\
\hline$\widehat{L}^{(128)}$ & 0.6241 & 0.0000265 \\
\hline$\widehat{L}^{(129)}$ & 0.6262 & 0.0000608 \\
\hline$\widehat{L}^{(130)}$ & 0.6283 & 0.0000485 \\
\hline$\widehat{L}^{(131)}$ & 0.6304 & 0.0010754 \\
\hline$\widehat{L}^{(132)}$ & 0.6325 & 0.0024652 \\
\hline$\widehat{L}^{(133)}$ & 0.6346 & 0.0000019 \\
\hline$\widehat{L}^{(134)}$ & 0.6367 & 0.0000416 \\
\hline$\widehat{L}^{(135)}$ & 0.6388 & 0.0000954 \\
\hline$\widehat{L}^{(136)}$ & 0.8600 & 0.0000108 \\
\hline$\widehat{L}^{(137)}$ & 0.8621 & 0.0002398 \\
\hline$\widehat{L}^{(138)}$ & 0.8642 & 0.0005497 \\
\hline$\widehat{L}^{(139)}$ & 0.8663 & 0.0004378 \\
\hline$\widehat{L}^{(140)}$ & 0.8684 & 0.0097171 \\
\hline$\widehat{L}^{(141)}$ & 0.8705 & 0.0222749 \\
\hline$\widehat{L}^{(142)}$ & 0.8726 & 0.0000169 \\
\hline$\widehat{L}^{(143)}$ & 0.8747 & 0.0003761 \\
\hline$\widehat{L}^{(144)}$ & 0.8768 & 0.0008622 \\
\hline$\widehat{L}^{(145)}$ & 0.8810 & 0.0001520 \\
\hline$\widehat{L}^{(146)}$ & 0.8831 & 0.0033738 \\
\hline$\widehat{L}^{(147)}$ & 0.8852 & 0.0077340 \\
\hline$\widehat{L}^{(148)}$ & 0.8873 & 0.0061597 \\
\hline$\widehat{L}^{(149)}$ & 0.8894 & 0.1367123 \\
\hline$\widehat{L}^{(150)}$ & 0.8915 & 0.3133927 \\
\hline$\widehat{L}^{(151)}$ & 0.8936 & 0.0002384 \\
\hline$\widehat{L}^{(152)}$ & 0.8957 & 0.0052917 \\
\hline$\widehat{L}^{(153)}$ & 0.8978 & 0.0121304 \\
\hline$\widehat{L}^{(154)}$ & 0.9020 & 0.0000057 \\
\hline$\widehat{L}^{(155)}$ & 0.9041 & 0.0001262 \\
\hline$\widehat{L}^{(156)}$ & 0.9062 & 0.0002893 \\
\hline$\widehat{L}^{(157)}$ & 0.9083 & 0.0002304 \\
\hline$\widehat{L}^{(158)}$ & 0.9104 & 0.0051131 \\
\hline$\widehat{L}^{(159)}$ & 0.9125 & 0.0117210 \\
\hline$\widehat{L}^{(160)}$ & 0.9146 & 0.0000089 \\
\hline$\widehat{L}^{(161)}$ & 0.9167 & 0.0001979 \\
\hline$\widehat{L}^{(162)}$ & 0.9188 & 0.0004537 \\
\hline
\end{tabular}

synthetically fused to derive the final evaluation result, which cannot be decided by a single criterion. In this process, any criterion cannot decide the evaluation result of MRES. Even though the information values on monitoring system and regular inspections $\left(c_{3}\right)$ and main biomass index $\left(c_{4}\right)$ are both definitely good, the final evaluation result is still influenced by three other criteria. Thus, the fusion of information at the criterion level is actually a process of tradeoff, which should follow compensatory strategies. Accordingly, we derive the holistic PLTSs (Table 7) by taking the criterion weight set $W=(0.150,0.050,0.100,0.400,0.300)$ and the normalized and ordered PLTSs as shown in Table 6 into equation (22).

As demonstrated in Table 7, values in the column of $\widehat{r}^{(k)}$ represent the subscripts of 162 virtual linguistic terms in the holistic PLTS, and values in the column of $\widehat{p}^{(k)}$ are their associated probabilities. Then, we apply the round function as in equation (11) to compute the integer score of the holistic PLTS and obtain $\alpha=0.670$. Then, we have $s^{*}=\operatorname{round}\left(s_{\alpha}\right)=s_{1}$. Thus, the ultimate evaluation result is that the MRES is evaluated as average.

\section{Conclusion}

MCGDM is a decision-making theory that has gained wide attention and applications. As a combination of MCDM and GDM, MCGDM contains two dimensions of information: individual and criterion. Distinction between these two kinds of information is important. Specifically, information at the individual level is noncompensatory in the process of fusion and influence brought by information with a lower degree of reliability cannot offset that brought by information with a higher degree of reliability. Information at the criterion level is compensatory in the process of fusion; the information offsets one another and jointly forms the fusion result. Unfortunately, existing MCGDM methods do not pay attention to this kind of distinction. Therefore, this paper proposes a novel MCGDM method with DST and PLTSs under the consideration of both compensatory and noncompensatory strategies. To conclude, this paper provides contributions in terms of the following aspects.

Firstly, this paper proposes that the information in the two dimensions in MCGDM is distinct, that is, information at the individual level is noncompensatory in the process of fusion, and information at the criterion level is compensatory. Accordingly, the proposed MCGDM method provides a comprehensive information fusion procedure that can fuse information at different levels.

Secondly, the proposed method provides a new orientation for the development of probabilistic preference theory in MCGDM by distinguishing noncompensatory information at the individual level and compensatory information at the criterion level. With the assistance of DST, PLTSs were developed to support information fusion and improve the scientific validity of the MCGDM.

Thirdly, this paper provides a method of building links between group BPA functions and PLTSs. Consequently, a framework based on belief and plausibility for transforming the certain point-valued probabilities assigned on power set 
of the frame of discernment into interval-valued probabilities associated with single linguistic terms is proposed, which also provides way of correlating BPA functions with PLTSs.

Finally, this paper contributes to improving the application of probabilistic preference theory for evaluating MRES. This paper provides a relative algorithm for the proposed method, which can be easily converted into a core algorithm of a decision system to promote its application. A case simulation study is also presented by applying the proposed method to evaluate the MRES.

It is worth noting that this paper just uses DST and PLTSs for reference to support noncompensatory and compensatory fusion, respectively. Other effective noncompensatory and compensatory modes could also be applied following the framework in this paper according to different problem characteristics. In the process of fusing compensatory information at the criterion level, the weights of the criteria are assumed to be known in advance. This paper does not address the way of determining the criteria weights. However, as the weights of the criteria influence the value of the virtual linguistic terms in the holistic PLTS a lot during the application of the weighted addition operation, they may lead to distortion of the fusion result. Therefore, the criteria weights should be determined carefully and an appropriate determination principle of criteria weights that suits the weighted addition operation of PLTSs in MCGDM is a worthy future research direction.

\section{Data Availability}

The data used to support the findings of this study are available from the corresponding author upon request.

\section{Conflicts of Interest}

The authors declare that there are no conflicts of interest regarding the publication of this paper.

\section{Acknowledgments}

This research was supported by the Major Program of National Social Science Foundation of China under grant no. 18ZDA055, the National Natural Science Foundation of China (NSFC) under grant nos. 71874167, 71804170, 71901199, and 71462022, the Fundamental Research Funds for the Central Universities under grant no. 202041005, and the Special Funds of Taishan Scholars Project of Shandong Province under grant no. tsqn20171205.

\section{References}

[1] C. W. Churchman Jr. and R. L. Ackoff, "An approximate measure of value," Journal of the Operations Research Society of America, vol. 2, no. 2, pp. 172-187, 1954.

[2] T. Koopmans, Activity Analysis of Production and Allocation, Wiley, Hoboken, NJ, USA, 1951.

[3] S. Fan, J. Zhang, E. Blanco-Davis, Z. Yang, and X. Yan, "Maritime accident prevention strategy formulation from a human factor perspective using Bayesian networks and topsis," Ocean Engineering, vol. 210, p. 107544, 2020.

[4] S. Komsiyah, R. Wongso, and S. W. Pratiwi, "Applications of the fuzzy ELECTRE method for decision support systems of cement vendor selection," Procedia Computer Science, vol. 157, pp. 479-488, 2019.

[5] H. Liao, L. Jiang, B. Lev, and H. Fujita, "Novel operations of pltss based on the disparity degrees of linguistic terms and their use in designing the probabilistic linguistic ELECTRE III method," Applied Soft Computing, vol. 80, pp. 450-464, 2019.

[6] H.-M. Lyu, W.-H. Zhou, S.-L. Shen, and A.-N. Zhou, "Inundation risk assessment of metro system using Ahp and TfnAhp in Shenzhen," Sustainable Cities and Society, vol. 56, Article ID 102103, 2020.

[7] R. Micale, C. M. La Fata, and G. La Scalia, "A combined interval-valued ELECTRE tri and topsis approach for solving the storage location assignment problem," Computers \& Industrial Engineering, vol. 135, pp. 199-210, 2019.

[8] Y. Zhang, Z. Xu, and H. Liao, "Water security evaluation based on the todim method with probabilistic linguistic term sets," Soft Computing, vol. 23, no. 15, pp. 6215-6230, 2019.

[9] H. Li, W. Wang, L. Fan, Q. Li, and X. Chen, "A novel hybrid MCDM model for machine tool selection using fuzzy dematel, entropy weighting and later defuzzification vikor," Applied Soft Computing, vol. 91, p. 106207, 2020.

[10] S.-f. Zhang, S.-y. Liu, and R.-h. Zhai, "An extended gra method for MCDM with interval-valued triangular fuzzy assessments and unknown weights," Computers \& Industrial Engineering, vol. 61, no. 4, pp. 1336-1341, 2011.

[11] G. Pineda, P. Jose, J. James, H. Liou, C.-C. Hsu, and Y.-C. Chuang, "An integrated MCDM model for improving airline operational and financial performance," Journal of Air Transport Management, vol. 68, pp. 103-117, 2018.

[12] A. Abdelli, L. Mokdad, and Y. Hammal, "Dealing with value constraints in decision making using MCDM methods," Journal of Computational Science, vol. 44, p. 101154, 2020.

[13] Ž. Stević, D. Pamučar, A. Puška, and P. Chatterjee, "Sustainable supplier selection in healthcare industries using a new MCDM method: measurement of alternatives and ranking according to compromise solution (marcos)," Computers \& Industrial Engineering, vol. 140, p. 106231, 2020.

[14] S. Barak and T. Mokfi, "Evaluation and selection of clustering methods using a hybrid group MCDM," Expert Systems with Applications, vol. 138, p. 112817, 2019.

[15] R. A. Krohling and V. C. Campanharo, "Fuzzy topsis for group decision making: a case study for accidents with oil spill in the sea," Expert Systems with Applications, vol. 38, no. 4, pp. 4190-4197, 2011.

[16] J. Pang, X. Guan, J. Liang, B. Wang, and P. Song, "MultiAttribute group decision-making method based on multigranulation weights and three-way decisions," International Journal of Approximate Reasoning, vol. 117, pp. 122-147, 2020.

[17] H. Karimi, M. Sadeghi-Dastaki, and M. Javan, "A fully fuzzy best-worst multi attribute decision making method with triangular fuzzy number: a case study of maintenance assessment in the hospitals," Applied Soft Computing, vol. 86, p. 105882, 2020.

[18] Y.-J. Wang, "Interval-valued fuzzy multi-criteria decisionmaking based on simple additive weighting and relative preference relation," Information Sciences, vol. 503, pp. 319335, 2019.

[19] F. Meng, S.-M. Chen, and S. Zhang, "Group decision making based on acceptable consistency analysis of interval linguistic 
hesitant fuzzy preference relations," Information Sciences, vol. 530, pp. 66-84, 2020.

[20] X. Tan, J. Zhu, and Y. Zhang, "A consensus reaching process with quantum subjective adjustment in linguistic group decision making," Information Sciences, vol. 533, pp. 150-168, 2020.

[21] J. Qu, X. Meng, X. Jiang et al., "Effective aggregation of expert opinions to inform environmental management: an integrated fuzzy group decision-making framework with application to cadmium-contaminated water treatment alternatives evaluation," Journal of Cleaner Production, vol. 209, pp. 834-845, 2019.

[22] J. Tang, S.-M. Chen, and F. Meng, "Heterogeneous group decision making in the setting of incomplete preference relations," Information Sciences, 2019.

[23] F. Zhu, P.-a. Zhong, and Y. Sun, "Multi-criteria group decision making under uncertainty: application in reservoir flood control operation," Environmental Modelling \& Software, vol. 100, pp. 236-251, 2018.

[24] X. Zhang, H. Liao, B. Xu, and M. Xiong, “A probabilistic linguistic-based deviation method for multi-expert qualitative decision making with aspirations," Applied Soft Computing, vol. 93, Article ID 106362, 2020.

[25] J.-B. Yang and D.-L. Xu, "Evidential reasoning rule for evidence combination," Artificial Intelligence, vol. 205, pp. 1-29, 2013.

[26] G. A. Shafer, A Mathematical Theory of Evidence, Princeton University Press, Princeton, NJ, USA, 1976.

[27] Q. Pang, H. Wang, and Z. Xu, "Probabilistic linguistic term sets in multi-attribute group decision making," Information Sciences, vol. 369, pp. 128-143, 2016.

[28] M. Zhou, X.-B. Liu, Y.-W. Chen, and J.-B. Yang, "Evidential reasoning rule for Madm with both weights and reliabilities in group decision making," Knowledge-Based Systems, vol. 143, pp. 142-161, 2018.

[29] B. Liu, Q. Zhou, R.-X. Ding, I. Palomares, and F. Herrera, "Large-scale group decision making model based on social network analysis: trust relationship-based conflict detection and elimination," European Journal of Operational Research, vol. 275, no. 2, pp. 737-754, 2019.

[30] H. Liao, X. Gou, Z. Xu, X. J. Zeng, and F. Herrera, "Hesitancy degree-based correlation measures for hesitant fuzzy linguistic term sets and their applications in multiple criteria decision making," Information Ences, vol. 508, pp. 275-292, 2020.

[31] M. Xue, C. Fu, and S.-L. Yang, "Group consensus reaching based on a combination of expert weight and expert reliability," Applied Mathematics and Computation, vol. 369, p. $124902,2020$.

[32] R. K. Bhattacharya, N. D. Chatterjee, and K. Das, "Sub-basin prioritization for assessment of soil erosion susceptibility in Kangsabati, a plateau basin: a comparison between MCDM and swat models," Science of the Total Environment, vol. 734, p. $139474,2020$.

[33] J. Xiao, X. Wang, and H. Zhang, "Managing classificationbased consensus in social network group decision making: an optimization-based approach with minimum information loss," Information Fusion, vol. 63, pp. 74-87, 2020.

[34] T. Elrod, R. D. Johnson, and J. White, "A new integrated model of noncompensatory and compensatory decision strategies," Organizational Behavior and Human Decision Processes, vol. 95, no. 1, pp. 1-19, 2004.

[35] M. E. Banihabib, F.-S. Hashemi-Madani, and A. Forghani, "Comparison of compensatory and non-compensatory multi criteria decision making models in water resources strategic management," Water Resources Management, vol. 31, no. 12, pp. 3745-3759, 2017.

[36] S. Luan and J. Reb, "Fast-and-Frugal trees as noncompensatory models of performance-based personnel decisions," Organizational Behavior and Human Decision Processes, vol. 141, pp. 29-42, 2017.

[37] L. Rothrock and J. Yin, Integrating Compensatory and Noncompensatory Decision-Making Strategies in Dynamic Task Environments, pp. 125-141, Springer, New York, NY, USA, 2008.

[38] C. D. Von Gunten, L. D. Scherer, and L. D. Scherer, "Selfother differences in multiattribute decision making: compensatory versus noncompensatory decision strategies," Journal of Behavioral Decision Making, vol. 32, no. 2, pp. 109-123, 2019.

[39] H. Liao, Z. Xu, and F. Herrera, "Applications of contemporary decision-making methods to the development of economy and technology," Technological and Economic Development of Economy, vol. 26, no. 3, pp. 546-548, 2020.

[40] P. Li and C. Wei, "An emergency decision-making method based on D-S evidence theory for probabilistic linguistic term sets," International Journal of Disaster Risk Reduction, vol. 37, Article ID 101178, 2019.

[41] X. Wu and H. Liao, "An approach to quality function deployment based on probabilistic linguistic term sets and oreste method for multi-expert multi-criteria decision making," Information Fusion, vol. 43, pp. 13-26, 2018.

[42] C. Huang, M. Lin, and R. Chen, "Probabilistic linguistic VIKOR method based on TODIM for reliable participant selection problem in mobile crowdsensing," in IEEE International Conference on Big Data and Cloud Computing (BdCloud), Xiamen, China, December 2019.

[43] M. Lin, Z. Chen, H. Liao, and Z. Xu, "ELECTRE II method to deal with probabilistic linguistic term sets and its application to edge computing," Nonlinear Dynamics, vol. 96, no. 3, pp. 2125-2143, 2019.

[44] M. Lin, C. Huang, Z. Xu, and R. Chen, "Evaluating IoT platforms using integrated probabilistic linguistic MCDM method," IEEE Internet of Things Journal, vol. 7, no. 11, pp. 11195-11208, 2020.

[45] H. A. Simon, Administrative Behavior: A Study of DecisionMaking Process in Administrative Organization, Macmillan, New York, NY, USA, 1947.

[46] J. K. Nuamah and Y. Seong, "A machine learning approach to predict human judgments in compensatory and noncompensatory judgment tasks," IEEE Transactions on $\mathrm{Hu}$ man-Machine Systems, vol. 49, no. 4, pp. 326-336, 2019.

[47] A. P. Dempster, "Upper and lower probabilities induced by a multivalued mapping," The Annals of Mathematical Statistics, vol. 38, no. 2, pp. 325-339, 1967.

[48] Y.-W. Du, S.-S. Wang, N. Yang, and W. Zhou, "Multiple attribute large-group decision-making method with incomplete information by considering expert's knowledge structure," Chinese Journal of Management Science, vol. 25, no. 12, pp. 167-178, 2017.

[49] T. Gudiyangada, Sepideh Tavakkoli Piralilou, K. Gholamnia, O. Ghorbanzadeh, and T. Blaschke, "Flood susceptibility mapping with machine learning, multi-criteria decision analysis and ensemble using Dempster Shafer theory," Journal of Hydrology, vol. 590, Article ID 125275, 2020.

[50] L. Wan, H. Li, Y. Chen, and C. Li, "Rolling bearing fault prediction method based on QPSO-BP neural network and Dempster-Shafer evidence theory," Energies, vol. 13, no. 5, p. 1094, 2020. 
[51] M. Mokarram, S. Ali, P. Mohammadizadeh, and A. Ali, "Determination of artificial recharge location using analytic hierarchy process and Dempster-Shafer theory," Environmental Earth Ences, vol. 79, p. 10, 2020.

[52] Y.-M. Wang, J.-B. Yang, D.-L. Xu, and K.-S. Chin, "The evidential reasoning approach for multiple attribute decision analysis using interval belief degrees," European Journal of Operational Research, vol. 175, no. 1, pp. 35-66, 2006.

[53] R. Haenni, "Shedding new light on Zadeh's criticism of Dempster's rule of combination," in Proceedings of the Paper Presented at the 2005 7th International Conference on Information Fusion, Philadelphia, PA, USA, July 2005.

[54] R. M. Rodriguez, L. Martinez, and F. Herrera, "Hesitant fuzzy linguistic term sets for decision making," IEEE Transactions on Fuzzy Systems, vol. 20, no. 1, pp. 109-119, 2012.

[55] F. Herrera, E. Herrera-Viedma, and J. L. Verdegay, "A sequential selection process in group decision making with a linguistic assessment approach," Information Sciences, vol. 85, no. 4, pp. 223-239, 1995.

[56] J. Gu, Y. Zheng, X. Tian, and Z. Xu, "A decision-making framework based on prospect theory with probabilistic linguistic term sets," Journal of the Operational Research Society, pp. 1-10, 2020.

[57] Y.-W. Du and X.-L. Sun, "Influence paths of marine ranching ecological security in China based on probabilistic linguistic term sets and qualitative comparative analysis," International Journal of Fuzzy Systems, 2020.

[58] Z. Xu and H. Wang, "On the syntax and semantics of virtual linguistic terms for information fusion in decision making," Information Fusion, vol. 34, pp. 43-48, 2017.

[59] Y. Zhang, Z. Xu, H. Wang, and H. Liao, "Consistency-based risk assessment with probabilistic linguistic preference relation," Applied Soft Computing, vol. 49, pp. 817-833, 2016.

[60] R. R. Yager, "Decision making under Dempster-Shafer uncertainties," International Journal of General Systems, vol. 20, no. 3, pp. 233-245, 1992.

[61] B. Suo, L. Zhao, and Y. Yan, "A novel Dempster-Shafer theorybased approach with weighted average for failure mode and effects analysis under uncertainty," Journal of Loss Prevention in the Process Industries, vol. 65, Article ID 104145, 2020.

[62] Y.-W. Du, S.-S. Wang, and Y.-M. Wang, "Group fuzzy comprehensive evaluation method under ignorance," Expert Systems With Applications, vol. 126, pp. 92-111, 2019.

[63] J. Yang and M. G. Singh, "An evidential reasoning approach for multiple-attribute decision making with uncertainty," IEEE Transactions on Systems, Man, and Cybernetics, vol. 24, no. 1, pp. 1-18, 1994.

[64] Z. Wang, J.-M. Gao, R.-X. Wang, K. Chen, Z.-Y. Gao, and Y. Jiang, "Failure mode and effects analysis using DempsterShafer theory and topsis method: application to the gas insulated metal enclosed transmission line (gil)," Applied Soft Computing, vol. 70, pp. 633-647, 2018.

[65] C. Bai, R. Zhang, S. Shen, C. Huang, and X. Fan, "Intervalvalued probabilistic linguistic term sets in multi-criteria group decision making," International Journal of Intelligent Systems, vol. 33, no. 6, pp. 1301-1321, 2018.

[66] P. Liu and S. Cheng, "Interval-valued probabilistic dual hesitant fuzzy sets for multi-criteria group decision-making," International Journal of Computational Intelligence Systems, vol. 2, no. 12, pp. 1393-1411, 2019.

[67] R. Krishankumar, A. R. Mishra, K. S. Ravichandran et al., "A group decision framework for renewable energy source selection under interval-valued probabilistic linguistic term set," Energies, vol. 13, no. 4, p. 986, 2020. 